\title{
Differentially expressed gene profile and relevant pathways of the traditional Chinese medicine cinobufotalin on MCF-7 breast cancer cells
}

\author{
JIE LI ${ }^{1}$, MIN-HUARONG ${ }^{2}$, YI-WU DANG ${ }^{3}$, RONG-QUAN HE ${ }^{4}$, PENG LIN ${ }^{5}$, \\ HONG YANG $^{5}$, XIAO-JIAO LI ${ }^{6}$, DAN-DAN XIONG ${ }^{3}$, LI-JIE ZHANG ${ }^{5}$, HUI QIN ${ }^{5}$, \\ CAI-XIA FENG ${ }^{3}$, XIAO-YI CHEN ${ }^{3}$, JIN-CAI ZHONG ${ }^{4}$, JIE MA $^{4}$ and GANG CHEN ${ }^{3}$
}

\begin{abstract}
${ }^{1}$ Department of Spleen and Stomach Diseases, The First Affiliated Hospital of Guangxi University of Traditional Chinese Medicine, Nanning, Guangxi Zhuang Autonomous Region 530023; ${ }^{2}$ Research Department,

The Affiliated Cancer Hospital, Nanning, Guangxi Zhuang Autonomous Region 530021; Departments of ${ }^{3}$ Pathology, ${ }^{4}$ Medical Oncology and ${ }^{5}$ Ultrasonics Division of Radiology; ${ }^{6}$ PET-CT, The First Affiliated Hospital of Guangxi Medical University, Nanning, Guangxi Zhuang Autonomous Region 530021, P.R. China
\end{abstract}

Received April 15, 2018; Accepted January 1, 2019

DOI: $10.3892 / \mathrm{mmr} .2019 .10062$

\begin{abstract}
Cinobufotalin is a chemical compound extracted from the skin of dried bufo toads that may have curative potential for certain malignancies through different mechanisms; however, these mechanisms remain unexplored in breast cancer. The aim of the present study was to investigate the antitumor mechanism of cinobufotalin in breast cancer by using microarray data and in silico analysis. The microarray data set GSE85871, in which cinobufotalin exerted influences on the MCF-7 breast cancer cells, was acquired from the Gene Expression Omnibus database, and the differentially expressed genes (DEGs) were analyzed. Subsequently, protein interaction analysis was conducted, which clarified the clinical significance of core genes, and Gene Ontology and Kyoto Encyclopedia of Genes and Genomes were used to analyze cinobufotalin-related pathways. The Connectivity Map (CMAP) database was used to select existing compounds that exhibited curative properties similar to those of cinobufotalin. A total of 1,237 DEGs were identified from breast cancer cells that were treated with cinobufotalin. Two core genes, SRC proto-oncogene non-receptor tyrosine kinase and cyclin-dependent kinase inhibitor $2 \mathrm{~A}$, were identified as serving a vital role in the onset and development of breast cancer, and their expression levels were markedly reduced following cinobufotalin treatment as detected by the microarray of GSE85871. It also was revealed
\end{abstract}

Correspondence to: Professor Jie Li, Department of Spleen and Stomach Diseases, The First Affiliated Hospital of Guangxi University of Traditional Chinese Medicine, 89-9 Dongge Road, Nanning, Guangxi Zhuang Autonomous Region 530023, P.R. China E-mail: 254926526@qq.com

Key words: gene profile, pathways, traditional Chinese medicine, cinobufotalin, breast cancer that the 'neuroactive ligand-receptor interaction' and 'calcium signaling' pathways may be crucial for cinobufotalin to perform its functions in breast cancer. Conducting a matching search in CMAP, miconazole and cinobufotalin were indicated to possessed similar molecular mechanisms. In conclusion, cinobufotalin may serve as an effective compound for the treatment of a subtype of breast cancer that is triple positive for the presence of estrogen, progesterone and human epidermal growth factor receptor- 2 receptors, and its mechanism may be related to different pathways. In addition, cinobufotalin is likely to exert its antitumor influences in a similar way as miconazole in MCF-7 cells.

\section{Introduction}

Breast cancer is one of the most common malignancies in women worldwide and results in relatively high rates of morbidity and mortality (1-4). In China, the incidence rate of breast cancer has been increasing over the past 20 years (5-7). Depending on the molecular classification, therapies used to treat breast cancer, including surgery, chemotherapy, radiation therapy, hormone (endocrine) therapy and molecule-targeted therapy, vary in their survival rates (8-13). As the prognosis for breast cancer patients remains unsatisfying (14-18), there is an urgent need to identify a more effective therapy.

Several chemical compounds used in traditional Chinese medicine (TCM) have proven useful in some conventional chemotherapies $(19,20)$. Cinobufotalin, a member of the bufadienolide family, is isolated from the skin parotoid glands of toads, such as Bufo gargarizans and Duttaphrynus melanostictus (21). The broad-spectrum antineoplastic activity and chemosensitization of bufadienolide has also been previously reported (22). Another study on cinobufotalin revealed that it may serve as a cardiotonic, diuretic and hemostatic agent (23). Previous reports have also focused on the influences of cinobufotalin on cancers such as hepatocellular carcinoma (HCC) and lung cancer $(24,25)$, but very few studies have examined 
its mechanism in these malignancies and there are even fewer, if any, reports on its functions in breast cancer. The mechanism of cinobufotalin against breast cancer cells remain unknown.

In the present study, the GSE85871 microarray data set from the Gene Expression Omnibus (GEO) database was used in an optimized analysis to identify differentially expressed genes (DEGs) in MCF-7 breast cancer treated with cinobufotalin. Subsequently, the potential molecular mechanism of cinobufotalin in breast cancer was explored through gene annotation, pathway analysis and protein-protein interaction (PPI) analysis. Connectivity Map (CMAP) analysis was used to identify drugs that may exhibit similar curative properties as cinobufotalin. Based on the mining of a large database, the present study comprehensively revealed the roles of cinobufotalin and its potential molecular mechanism in breast cancer, and offered a possible avenue for breast cancer treatment.

\section{Materials and methods}

Data sources and analysis of DEG expression profiles. The expression data of the GSE85871 data set were obtained from the National Center for Biotechnology Information GEO database (26). The subject of this microarray was Homo sapiens, and its research type was expression profiling by array. The expression profile of this microarray was provided by GPL571 (HG-U133A_2; Affymetrix Human Genome U133A 2.0 Array). GSE85871 included the gene expression profiles of 102 TCM compounds used to treat MCF-7 cells triple positive for the presence of estrogen, progesterone and human epidermal growth factor receptor-2 (HER-2) receptors. Additionally, in this microarray, comparisons were made between the experimental groups treated with each drug and their respective dimethylsulphoxide (DMSO) controls; there were two expression values for each drug. The expression profiles of genes in MCF-7 cells treated with cinobufotalin (GSM2286314 MCF-7_Cinobufotalin_1 $\mu \mathrm{M}$ _rep1 and GSM2286315 MCF-7_Cinobufotalin_1 $\left.\mu \mathrm{M} \_r e p 2\right)$ and the profiles of respective controls [GSM2286316 MCF-7_vehicle (DMSO)_rep1 and GSM2286317 MCF-7_vehicle (DMSO)_rep2] were downloaded from the GSE85871 data set. Fold change (FC) was set as the threshold for the mean value of gene expression in the experimental groups and in the respective DMSO controls, as previously described (26); DEGs were identified as $\mathrm{FC} \geq 2$ or $\mathrm{FC} \leq 0.5$, and categorized as upregulated or downregulated, respectively.

Functions of DEGs and pathway enrichment analysis. The STRING online tool (https://string-db.org) was used for a PPI network analysis. The top 10 genes that possessed the most protein interactions were considered hub genes, and their genetic alterations were displayed using The Cancer Genome Atlas (TCGA; https://cancergenome.nih.gov) data calculated with the cBioPortal $(27,28)$. The cBioPortal dataset included 3 different data: TCGA RNA-sequencing, Provisional; Genomic Identification of Significant Targets In Cancer (GISTIC) data set; and, a microarray included in the TCGA project. mRNA and protein expression levels and clinical significance of these hub genes were computed by Gene Expression Profiling Interactive Analysis (GEPIA) and The Human Protein Atlas (29-34); TCGA and The Genotype-Tissue Expression
(GTEx) RNA-sequencing data were included in GEPIA. The expression levels of genes of interest were extracted from both TCGA and GTEx projects. Transcripts Per Kilobase Million (TPM) was used to calculate mRNA expression levels, and the relative expression levels of genes were presented as $\log _{2}(T P M+1)$. Student's t-test was used to compare the differences in expression between cancer and non-cancerous tissues; $\mathrm{P}<0.05$ was considered to indicate a statistically significant difference. Gene Ontology (GO; http://www.geneontology. org) and Kyoto Encyclopedia of Genes and Genomes (KEGG; https://www.genome.jp/kegg) pathway analyses were carried out for DEGs post-cinobufotalin treatment of breast cancer cells in GSE85871 through DAVID (https://david.ncifcrf. gov) (35-39). Cytoscape (https://cytoscape.org) and R software were used to display the top 10 terms (40-45).

CMAP matching of cinobufotalin with existing compounds. The CMAP database (https://clue.io/cmap) was used to further investigate the relationship between the effectiveness of cinobufotalin on treating breast cancer cells and to explore its pharmacology and mechanism. The enrichment value in CMAP represented a similarity in gene mapping. A value close to 1 indicated a positive relationship between two drug molecules, which suggested that cinobufotalin exhibited similar curative properties to the existing compounds. Conversely, a negative value indicated that cinobufotalin exhibited opposite functions to the existing compounds (46-48). The molecular structures of certain compounds were retrieved from PubChem (https://pubchem.ncbi.nlm.nih.gov/substance), including cinobufotalin (PubChem CID: 259776; Fig. 1).

\section{Results}

Analysis of DEGs and pathway enrichment. A total of 1,237 DEGs were identified in cinobufotalin-treated MCF-7 cells compared with the DMSO-treated controls. Of these, 641 genes were upregulated and 596 were downregulated (Fig. 2). To determine the protein interactions of these 1,237 DEGs, a PPI network was constructed using STRING (Fig. 3). PPI network analysis revealed several hub genes, including: Albumin (ALB); SRC proto-oncogene non-receptor tyrosine kinase (SRC); glucagon; protein tyrosine phosphatase receptor type $\mathrm{C}$ (PTPRC); spectrin $\alpha$ non-erythrocytic 1; coagulation factor II, thrombin; FYN proto-oncogene, SRC family tyrosine kinase (FYN); cyclin-dependent kinase inhibitor 2A (CDKN2A); transferrin (TF) and insulin-like growth factor 1 . The associations between these 10 genes are demonstrated in Fig. 4. As these genes could be the targets of cinobufotalin treatment in breast cancer cells, the clinical roles of these genes in breast cancer were next investigated using various approaches. Through cBioPortal data mining (Fig. 5), varied genetic alterations were observed in these genes in clinical breast cancer tissue samples, including amplification, a number of different mutations, and changes in mRNA and protein expression levels detected by multiple approaches. These genetic alterations were identified by three independent sources as provided by cBioPortal, including TCGA RNA-sequencing, Provisional (Fig. 5A); GISTIC data set (Fig. 5B) and a microarray included in the TCGA project (Fig. 5C). Based on the TCGA data, the amplification of ALB, SRC and PTPRC were the main genetic alteration events 
A

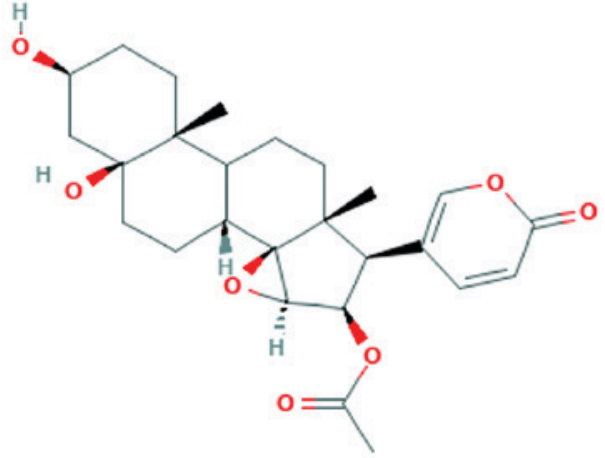

B

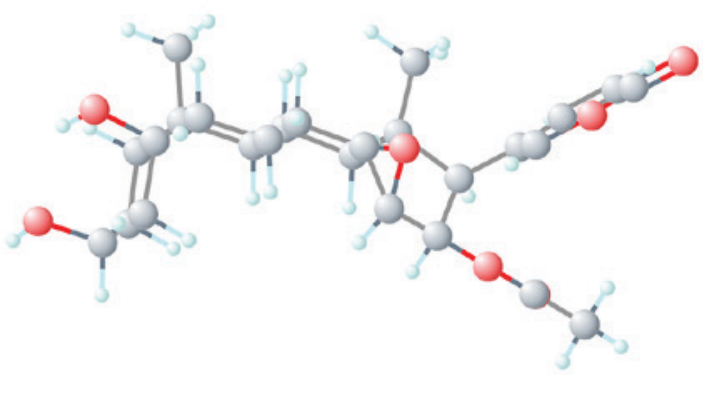

Figure 1. Molecular structure of cinobufotalin. Cinobufotalin chemical structure obtained from PubChem; PubChem CID: 259776. (A) 2D molecular structure. (B) 3D molecular structure.

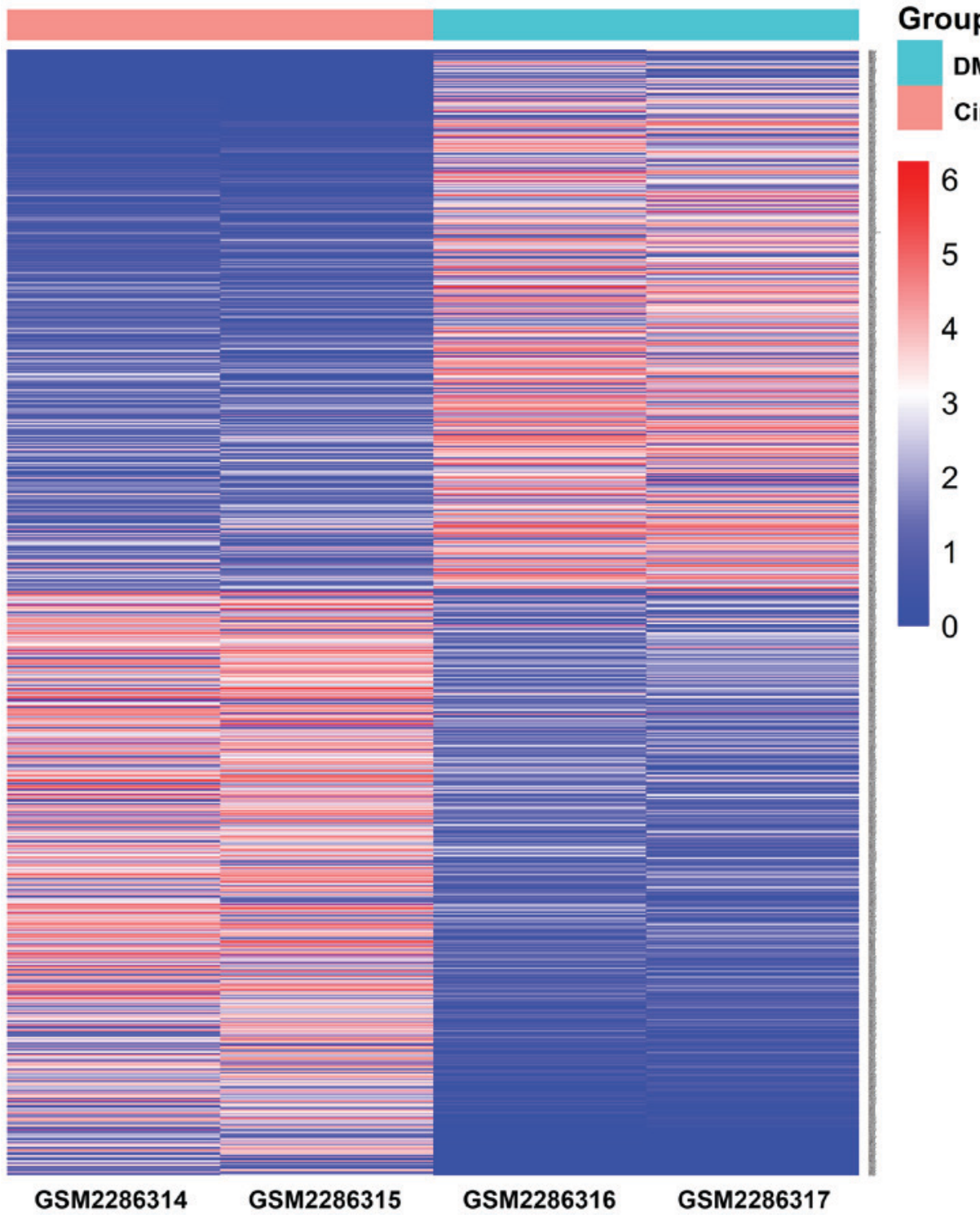

Figure 2. DEG microarray analysis in MCF-7 cells treated with cinobufotalin. A total of 1,237 DEGs were identified between cinobufotalin-treated and DMSO-treated MCF-7 breast cancer cells, of which 641 genes were upregulated and 596 were downregulated using cut-off values set as FC $\geq 2$ or FC $\leq 0.5$, respectively. DEGs, differentially expressed genes; DMSO, dimethylsulphoxide.

identified (Fig. 5A). In addition, the corresponding mRNA and protein expression levels of ALB, FYN and TF tended to decrease in clinical breast cancer tissue samples (Fig. 6). The
mRNA expression levels of ALB, FYN and TF were predominantly downregulated based on RNA-sequencing data with 1,085 cases of breast cancer and 291 non-cancerous breast 


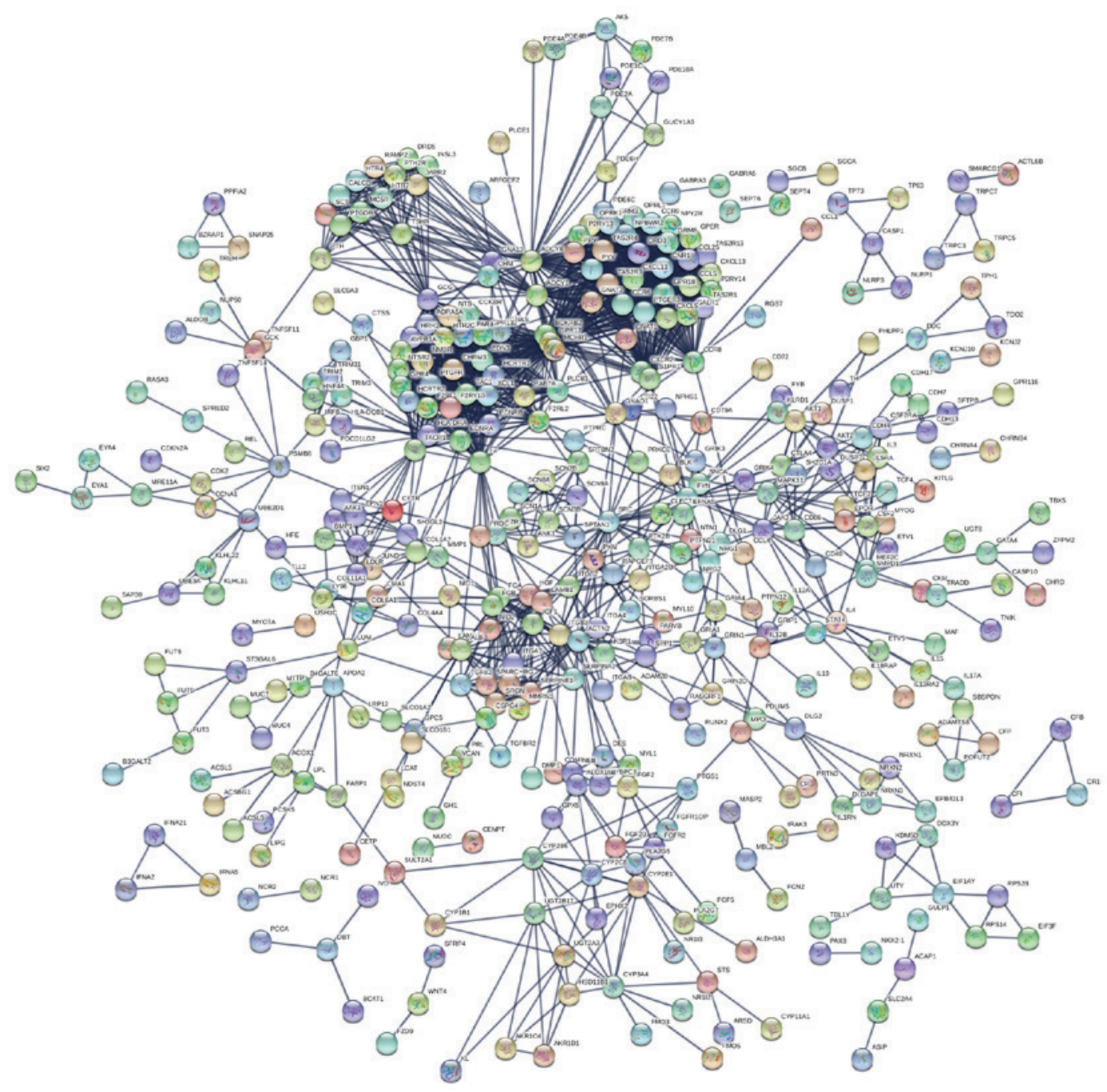

Figure 3. PPI network. A PPI network was created for the identified differentially expressed genes between MCF-7 breast cancer cells treated with cinobufotalin. Network nodes represent proteins; edges represent protein-protein associations. PPI, protein-protein interaction.

tissues (all $\mathrm{P}<0.05$ ), and the protein expression levels of ALB, FYN and TF were also decreased in breast cancer compared with the non-cancerous control tissues. The mRNA and protein expression levels of SRC and CDKN2A were increased in breast cancer tissue samples, compared with the control tissue (Fig. 7). However, owing to the limited number of cases provided by The Human Protein Atlas, statistical analysis was not possible. The protein expression levels of the above genes need to be confirmed using a larger cohort. Notably, following treatment with cinobufotalin, their expressions were remarkably reduced, with CDKN2A expression dropping to $42.2 \%$ and SRC plunging to $7.03 \%$ (data not shown). These results indicated that cinobufotalin is more likely to target these genes to exert its antitumor influences.

To explore the functions of these 1,237 DEGs, GO and KEGG analyses were conducted using DAVID. In the GO analysis, the genes were divided into three groups: i) Biological process (BP), ii) cellular component (CC) and iii) molecular function (MF). In BP, the three most significantly enriched processes were 'immune response', 'chemical synaptic transmission' and 'cellular defense response' (Fig. 8; Table IA). In $\mathrm{CC}$, the three most significant cellular components were 'integral component of plasma membrane', 'extracellular

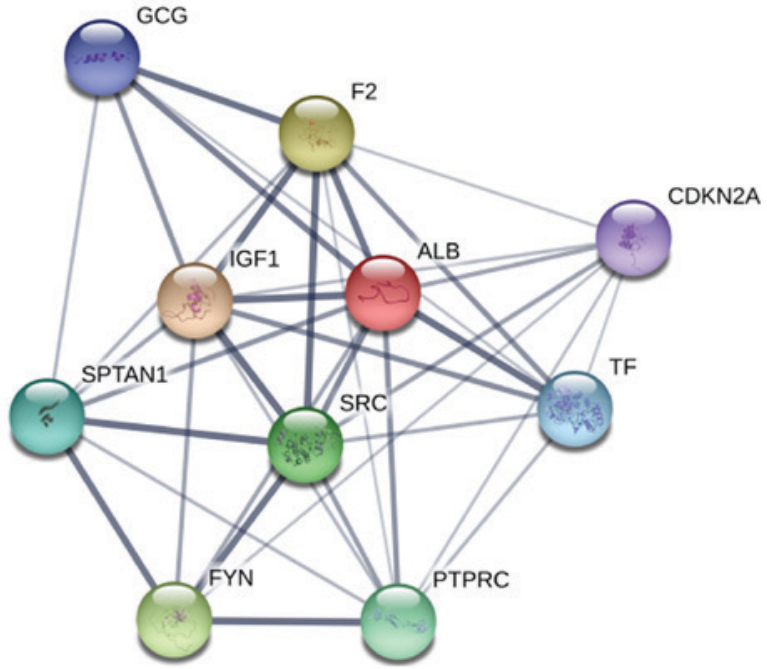

Figure 4. Protein-protein interaction network of the 10 hub genes in cinobufotalin-treated MCF-7 cells. Network nodes represent proteins; edges represent protein-protein associations. ALB, albumin; CDKN2A, cyclin-dependent kinase inhibitor $2 \mathrm{~A}$; GCG, glucagon; F2, coagulation factor II, thrombin; FYN, FYN proto-oncogene, SRC family tyrosine kinase; IGF1, insulin-like growth factor 1; PTPRC, protein tyrosine phosphatase receptor type C; SPTAN1, spectrin $\alpha$ non-erythrocytic 1; SRC, SRC proto-oncogene non-receptor tyrosine kinase; TF, transferrin. 
A Altered in $276(25 \%)$ of 1098 sequenced cases/patients (1098 total)

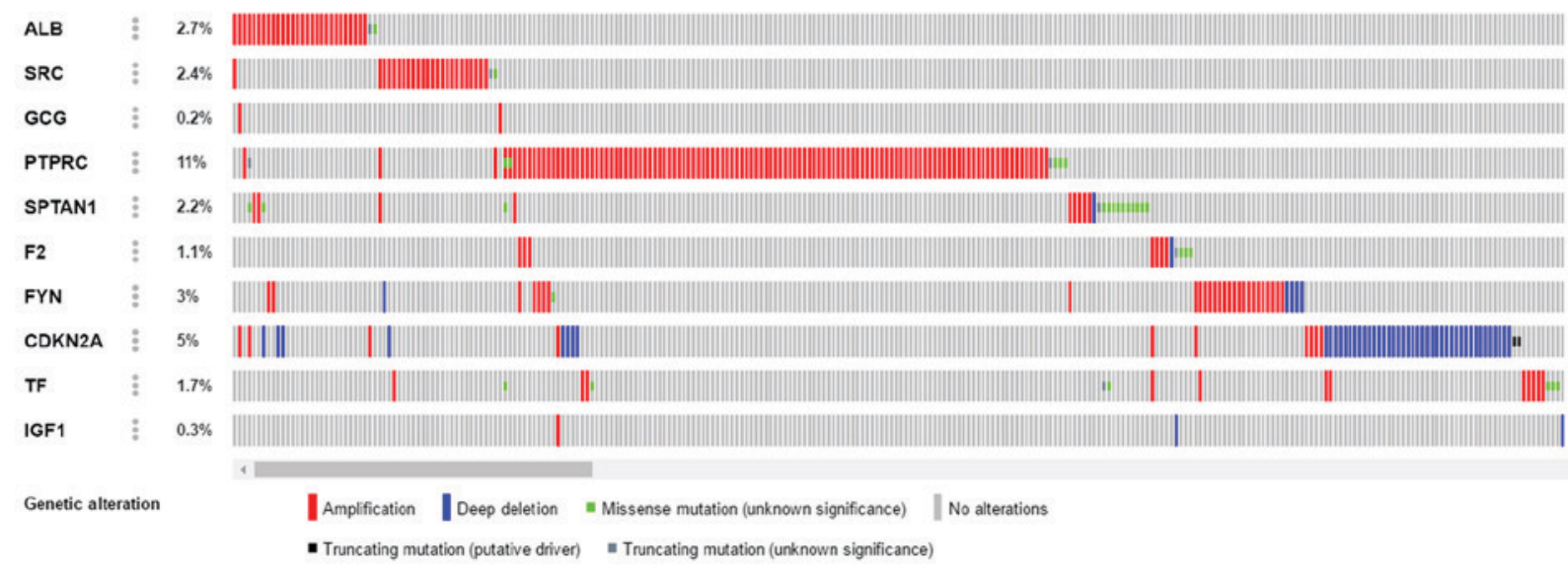

B Altered in $449(41 \%)$ of 1098 sequenced cases/patients (1098 total)

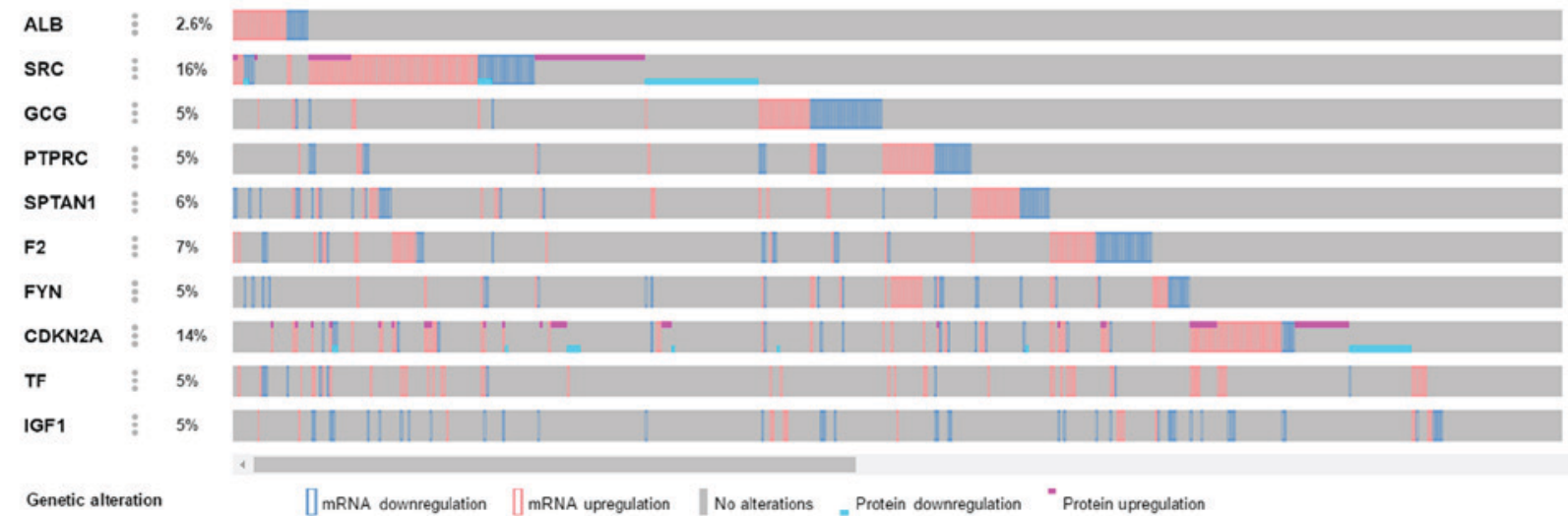

C Altered in $482(44 \%)$ of 1098 sequenced cases/patients (1098 total)

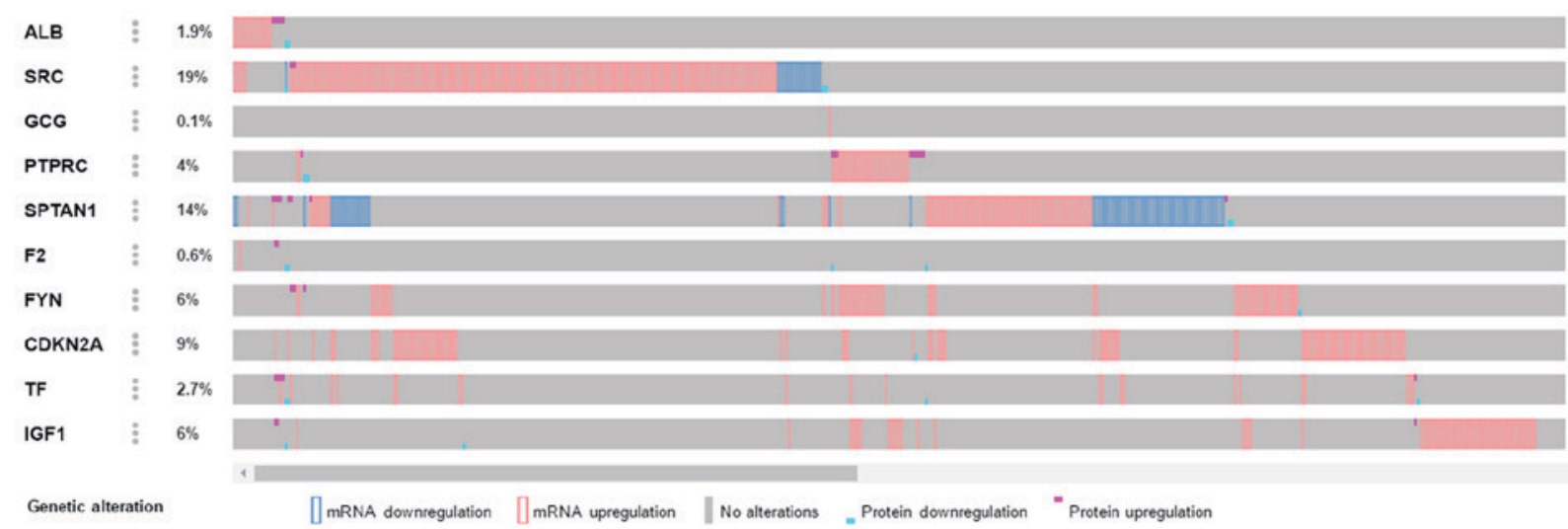

Figure 5. Genetic alterations of the 10 hub genes in breast cancer tissue samples identified in cinobufotalin-treated breast cancer tissues. The genetic alterations were from cBioPortal comprising 1,105 cases of breast cancer tissues (TCGA, Provisional). (A) Mutations and putative copy-number alterations from Genomic Identification of Significant Targets In Cancer from the TCGA project. (B) mRNA expression Z-Score (a microarray from TCGA project), protein/phosphoprotein level Z-Score, threshold=1.5. (C) mRNA expression Z-Score (RNA Seq V2 RSEM from TCGA project), protein/phosphoprotein level Z-Score (mass spectrometry by CPTAC), threshold=1.5. ALB, albumin; CDKN2A, cyclin-dependent kinase inhibitor 2A; GCG, glucagon; F2, coagulation factor II, thrombin; FYN, FYN proto-oncogene, SRC family tyrosine kinase; IGF1, insulin-like growth factor 1; PTPRC, protein tyrosine phosphatase receptor type C; SPTAN1, spectrin $\alpha$ non-erythrocytic 1; SRC, SRC proto-oncogene non-receptor tyrosine kinase; TCGA, The Cancer Genome Atlas; TF, transferrin.

space' and 'plasma membrane' (Fig. 9; Table IB). In MF, the top three functions were 'receptor binding', 'immunoglobulin receptor binding' and 'cytokine activity' (Fig. 10; Table IC). KEGG pathway analysis confirmed that DEGs were remarkably enriched in pathways of 'neuroactive ligand-receptor interaction', 'calcium signaling pathway' and 'steroid hormone biosynthesis' (Fig. 11; Table ID). The top five GO terms for each category and KEGG pathways concerning cinobufotalin treatment on breast cancer cells in GSE85871 are displayed in Table I.

Results of CMAP analysis. A gene expression profile comparison was conducted using the aforementioned 1,237 DEGs post-cinobufotalin treatment and those genes related to the known drugs from the whole CMAP database and 9 compounds yielded scores $>0.962$ (Table II), including 
A

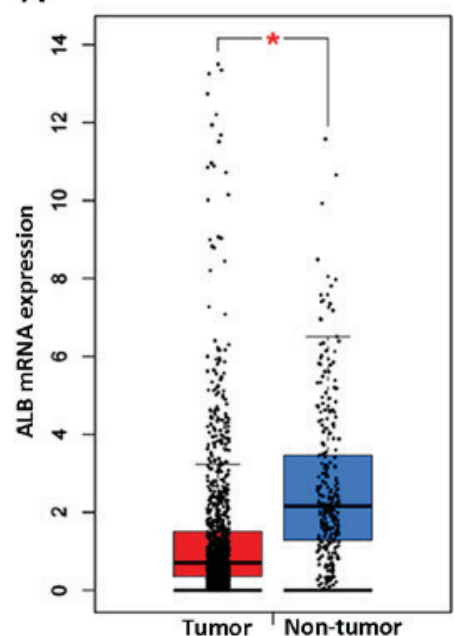

D

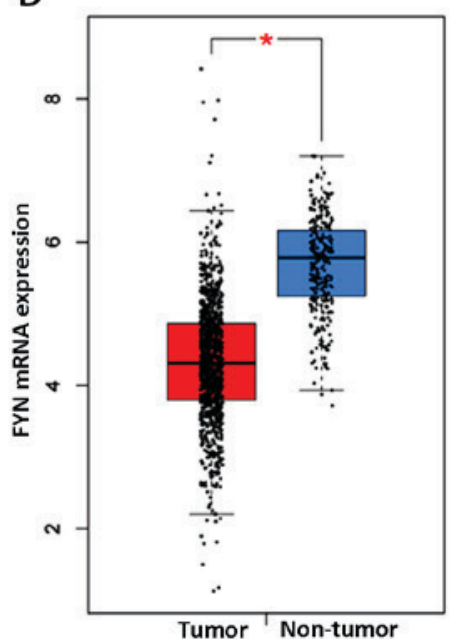

G

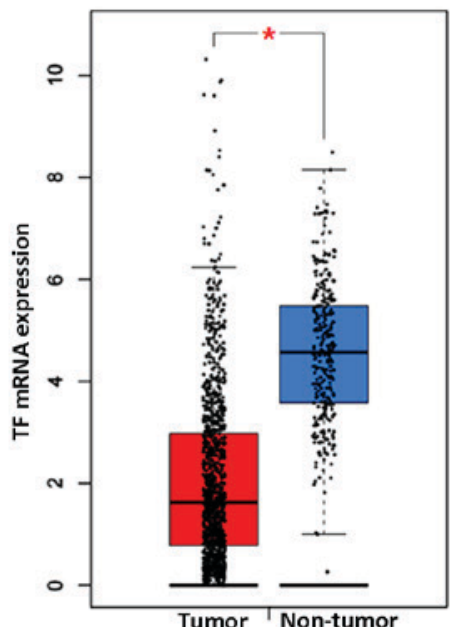

B

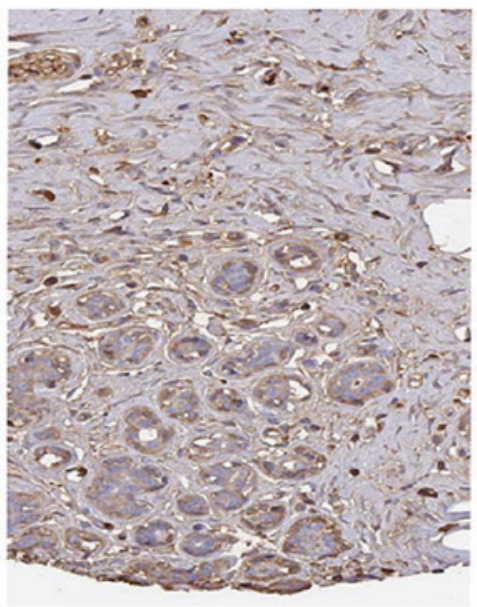

$\mathrm{E}$

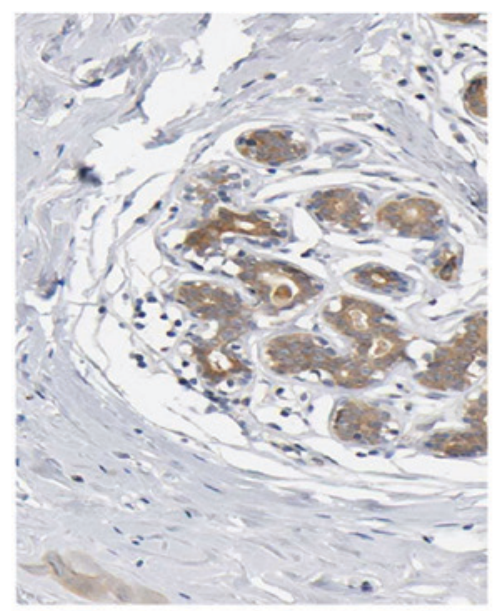

$\mathrm{H}$

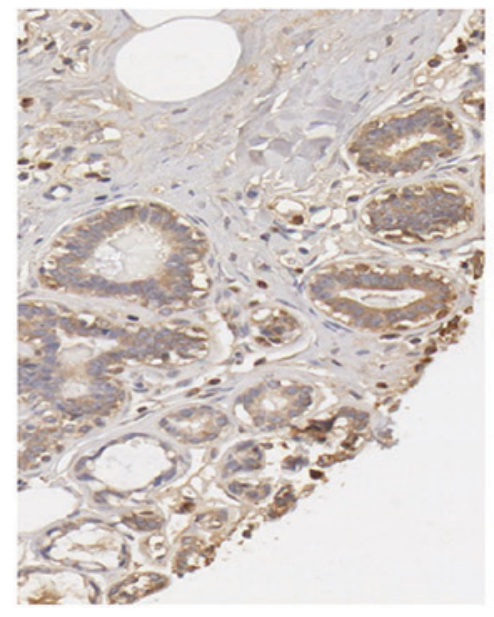

C

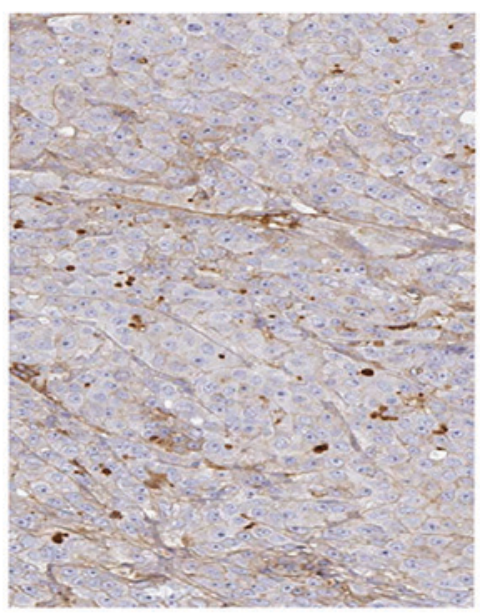

$\mathrm{F}$

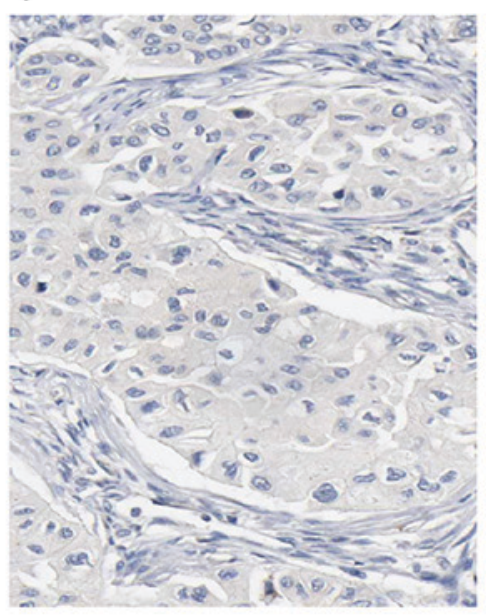

I

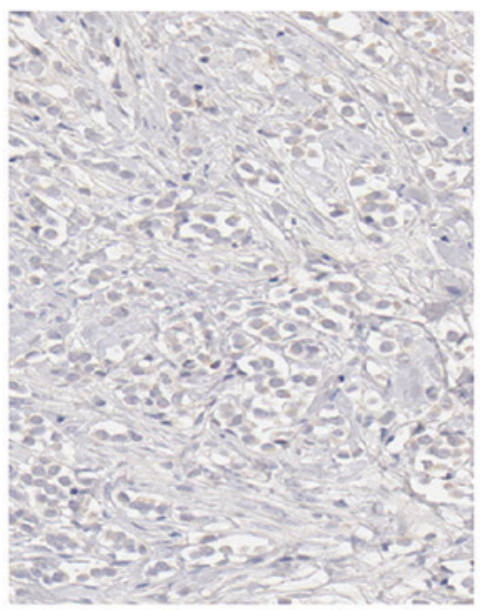

Figure 6. mRNA and protein expressions of ALB, FYN and TF in breast cancer tissues. (A-I) The GEPIA database, which included TCGA and GTEx RNA-sequencing data, was used to examine (A-C) ALB, (D-F) FYN and (G-I) TF mRNA and protein expression in breast cancer tissues and non-cancerous breast tissues; TPM was used to calculate relative mRNA gene expression levels from 1,085 tumor and 291 non-tumor tissues, which are presented as $\log _{2}($ TPM + 1) scale. (A) ALB mRNA expression levels from GEPIA. (B) Non-cancerous breast myoepithelial cells stained with HPA031024 anti-ALB antibody (medium staining). (C) Breast cancer tissue stained with HPA031024 anti-ALB antibody (low staining with light brown color in the cytoplasm). (D) FYN mRNA expression levels from GEPIA. (E) Non-cancerous breast myoepithelial cells stained with anti-FYN HPA023887 antibody (medium staining). (F) Breast cancer tissue stained with anti-FYN HPA023887 antibody (low staining with light brown color in the cytoplasm). (G) TF mRNA expression levels from GEPIA. (H) Non-cancerous breast myoepithelial cells stained with HPA001527 anti-TF antibody (medium staining). (I) Breast cancer tissue stained with HPA001527 anti-TF antibody (low staining with light brown color in the cytoplasm). ${ }^{*} \mathrm{P}<0.01$. Immunohistochemistry images in B, C, E, F, H and I are presented at x400 magnification. ALB, albumin; FYN, FYN proto-oncogene, SRC family tyrosine kinase; GEPIA, Gene Expression Profiling Interactive Analysis database; GTEx, Genotype-Tissue Expression; TCGA, The Cancer Genome Atlas; TF, transferrin; TPM, transcripts per kilobase million. 
A

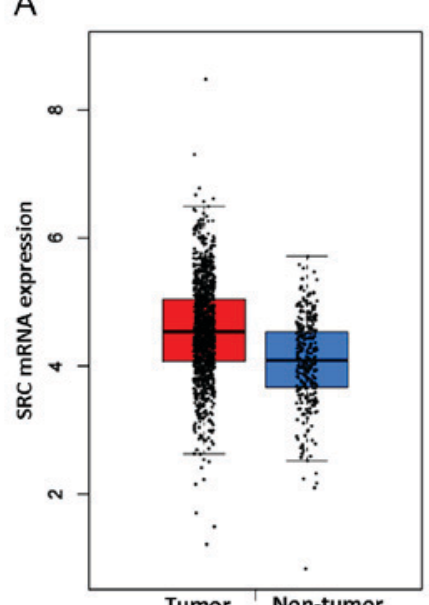

D

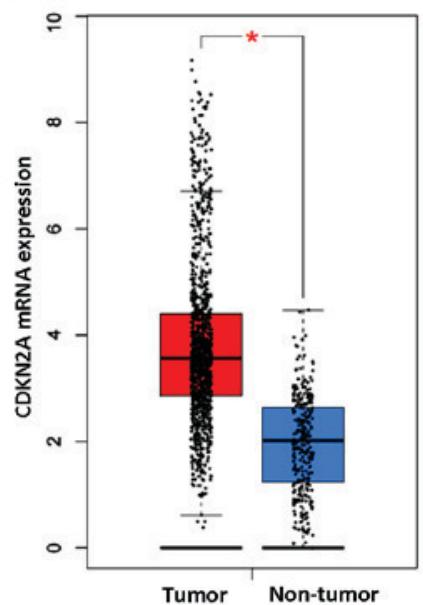

B

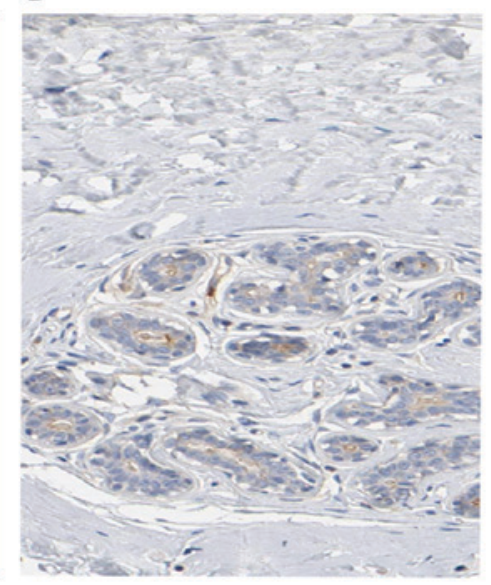

$\mathrm{E}$

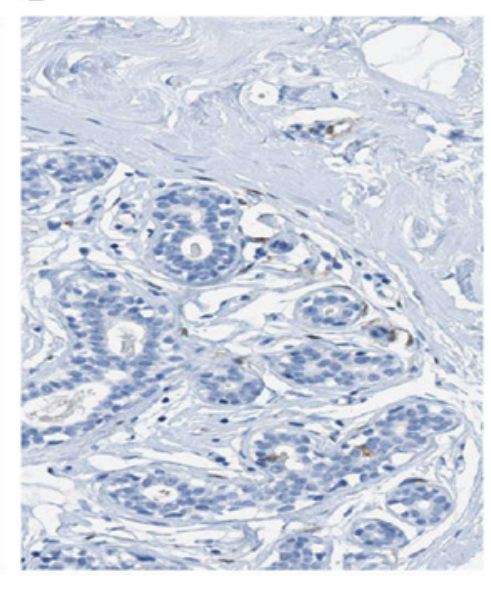

C

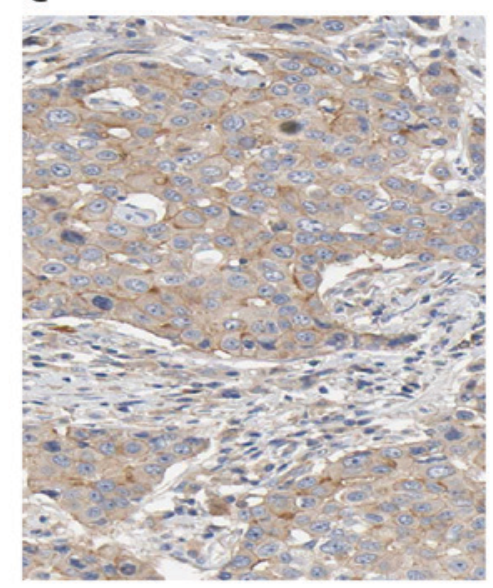

$\mathrm{F}$

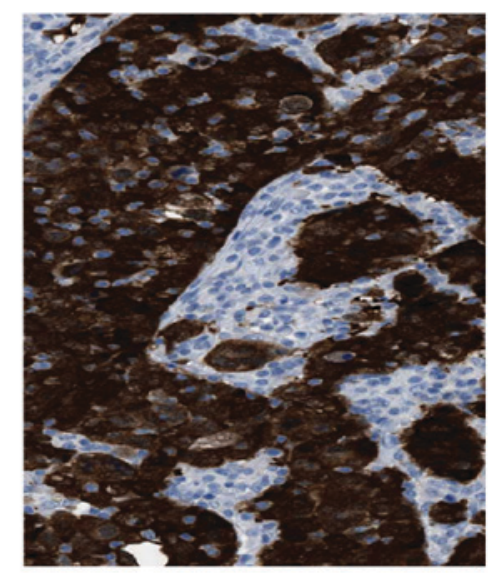

Figure 7. mRNA and protein expressions of SRC and CDKN2A in breast cancer tissues. (A-F) The GEPIA database, which included TCGA and GTEx RNA-sequencing data, was used to examine (A-C) SRC and (D-F) CDKN2A mRNA and protein expression in breast cancer tissues and non-cancerous breast tissues; TPM was used to calculate relative mRNA gene expression levels from 1,085 tumor and 291 non-tumor tissues, which are presented as $\log _{2}(\mathrm{TPM}+1)$ scale. (A) SRC mRNA expression levels from GEPIA. (B) Non-cancerous breast myoepithelial cells stained with CAB004023 anti-SRC antibody (not detected; brown staining was in the lumen of the ducts, not in the myoepithelial cells. (C) Breast cancer tissue stained with CAB004023 anti-SRC antibody (medium staining). (D) CDKN2A mRNA expression levels from GEPIA. (E) Non-cancerous breast myoepithelial cells stained with CAB018232 anti CDKN2A antibody (not detected). (F) Breast cancer tissue stained with CAB018232 anti CDKN2A antibody (high staining). * $<<0.01$. Immunohistochemistry images in B, C, E and F are presented at x400 magnification. CDKN2A, cyclin-dependent kinase inhibitor 2A; GEPIA, Gene Expression Profiling Interactive Analysis database; GTEx, Genotype-Tissue Expression; SRC, SRC proto-oncogene non-receptor tyrosine kinase; TCGA, The Cancer Genome Atlas; TPM, transcripts per kilobase million.

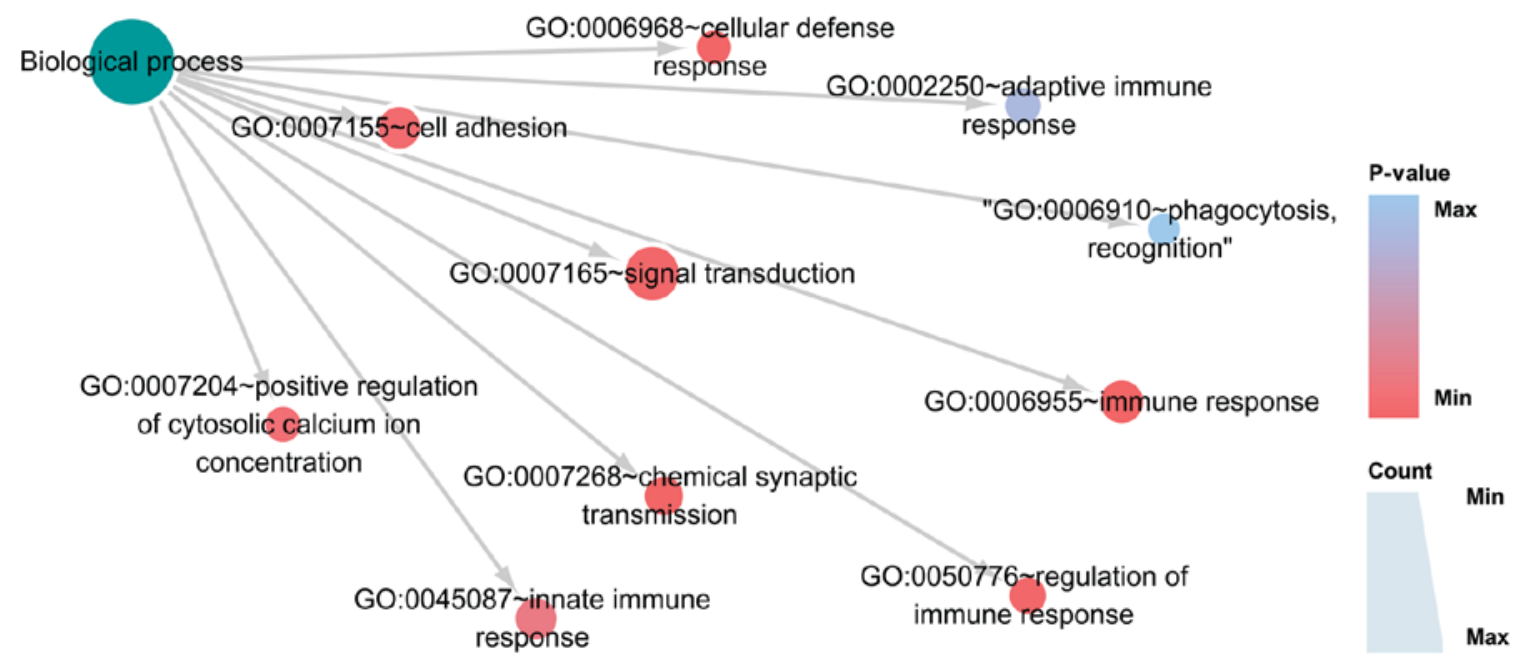

Figure 8. Biological process of differentially expressed genes in MCF-7 cells treated with cinobufotalin. Circles represent different GO terms; colors of circles indicate the significance of the corresponding terms, and the sizes of circles display the numbers of genes enriched in this term. GO, Gene Ontology. 
Table I. Significant terms of GO annotation and KEGG pathway enrichment analysis of cinobufotalin in GSE85871.

A, Biological process ${ }^{\mathrm{a}}$

\begin{tabular}{|c|c|c|c|c|}
\hline ID & Term & Count (\%) & P-value & Genes \\
\hline GO:0006955 & Immune response & $65(5.36)$ & $1.15 \times 10^{-11}$ & $\begin{array}{l}\text { IGLV1-44, IL16, IL19, TRGC2, TNFSF14, } \\
\text { IL15, ZEB1, CXCL11, CFP and CD96 }\end{array}$ \\
\hline GO:0007268 & $\begin{array}{l}\text { Chemical synaptic } \\
\text { transmission }\end{array}$ & $44(6.36)$ & $1.63 \times 10^{-10}$ & $\begin{array}{l}\text { SLC6A2, OPRK1, TACR1, NPY2R, DRD5, } \\
\text { GRIK4, KCNA1, CACNB1, TAC1 and } \\
\text { PMCHL1 }\end{array}$ \\
\hline GO:0006968 & $\begin{array}{l}\text { Cellular defense } \\
\text { response }\end{array}$ & $21(7.36)$ & $3.51 \times 10^{-10}$ & $\begin{array}{l}\text { IL4, NCF1, LY96, IL1RL2, CD160, GAGE2D, } \\
\text { GAGE2E, CXCR2, UMOD and KIR2DS3 }\end{array}$ \\
\hline GO:0007165 & Signal transduction & $121(8.36)$ & $6.20 \times 10^{-09}$ & $\begin{array}{l}\text { GNA13, EDN3, ADCY8, IL19, TRGC2, } \\
\text { RRAD, IQGAP2, TNFSF14, IL15 and } \\
\text { CXCL11 }\end{array}$ \\
\hline GO:0050776 & $\begin{array}{l}\text { Regulation of immune } \\
\text { response }\end{array}$ & $34(9.36)$ & $9.12 \times 10^{-09}$ & $\begin{array}{l}\text { IGLV1-44, KLRK1, ITGB2, CD96, SH2D1A, } \\
\text { IGHV3-23, IGLV6-57, KLRF1, IGKC and } \\
\text { KLRD1 }\end{array}$ \\
\hline
\end{tabular}

B, Cellular component ${ }^{\mathrm{a}}$

\begin{tabular}{llcll}
\hline ID & \multicolumn{1}{c}{ Term } & Count $(\%)$ & P-value & \multicolumn{1}{c}{ Genes } \\
\hline GO:0005887 & $\begin{array}{l}\text { Integral component of } \\
\text { plasma membrane } \\
\text { Extracellular space }\end{array}$ & $186(15.33)$ & $6.73 \times 10^{-27}$ & $\begin{array}{l}\text { F2RL2, ADCY1, SLC22A13, SLC6A2, GRIK4, } \\
\text { F2RL1, CSPG4, LPAR4 and TRGC2 }\end{array}$ \\
GO:0005886 & Plasma membrane & $365(17.33)$ & $1.20 \times 10^{-19}$ & $\begin{array}{l}\text { ADCY1, CROCC, SCN3B, ADCY8, SLC9A3, } \\
\text { SNCA, SLC9A2, LPAR4, AQP4 and PVRIG }\end{array}$ \\
GO:0005576 & Extracellular region & $181(18.33)$ & $1.34 \times 10^{-18}$ & $\begin{array}{l}\text { F2RL2, EDN3, IL16, IGLV1-44, MASP2, } \\
\text { IL19, SNCA, CSPG4, IL15 and MMRN1 }\end{array}$ \\
GO:0009897 & $\begin{array}{l}\text { External side of plasma } \\
\text { membrane }\end{array}$ & $39(19.33)$ & $5.71 \times 10^{-10}$ & $\begin{array}{l}\text { IGHG1, IGHG2, IGHG3, IGHG4, LDLR, } \\
\text { FCER2, KLRK1, AQP4, HFE and IGHM }\end{array}$ \\
\hline
\end{tabular}

C, Molecular function ${ }^{\mathrm{a}}$

\begin{tabular}{|c|c|c|c|c|}
\hline ID & Term & Count (\%) & P-value & Genes \\
\hline GO:0005102 & Receptor binding & $51(4.20)$ & $9.76 \times 10^{-09}$ & $\begin{array}{l}\text { INSL3, MBL2, EDN3, ACOX1, PPY, } \\
\text { PLXNC1, BLK, F2RL1, HFE and TAC1 }\end{array}$ \\
\hline GO:0034987 & $\begin{array}{l}\text { Immunoglobulin } \\
\text { receptor binding }\end{array}$ & $11(5.20)$ & $1.18 \times 10^{-06}$ & $\begin{array}{l}\text { IGHG1, IGHG2, IGHG3, IGHG4, IGHD, } \\
\text { IGHV3-23, IGHA1, IGHA2, IGKC, IGHM, } \\
\text { IGLC1 }\end{array}$ \\
\hline GO:0005125 & Cytokine activity & $29(6.20)$ & $1.78 \times 10^{-06}$ & $\begin{array}{l}\text { CSF2, IFNA21, IL16, IL19, KITLG, TNFSF14, } \\
\text { IL15, BMP15, CCL4 and FLT3LG }\end{array}$ \\
\hline GO:0003823 & Antigen binding & $21(7.20)$ & $2.38 \times 10^{-06}$ & $\begin{array}{l}\text { IGHG1, IGHG2, IGHG3, IGHG4, IGLV1-44, } \\
\text { HFE, CD40, ITGA4, IGHM and LILRA2 }\end{array}$ \\
\hline GO:0004872 & Receptor activity & $32(8.20)$ & $5.21 \times 10^{-06}$ & $\begin{array}{l}\text { NRP2, LDLR, NPY2R, F2RL1, KLRK1, } \\
\text { ITGB2, ITGB3, TLR8, LILRA2 and LILRA3 }\end{array}$ \\
\hline
\end{tabular}

D, KEGG pathway ${ }^{\mathrm{a}}$

\begin{tabular}{llcll}
\hline ID & Term & Count $(\%)$ & P-value & Genes \\
\hline hsa04080 & $\begin{array}{l}\text { Neuroactive ligand } \\
\text { receptor interaction }\end{array}$ & $61(5.03)$ & $2.65 \times 10^{-14}$ & $\begin{array}{l}\text { CSH1, F2RL2, MCHR1, CSH2, GRIK3, TACR1, } \\
\text { GRIK4, F2RL1, GLRA2 and LPAR4 }\end{array}$
\end{tabular}


Table I. Continued.

D, KEGG pathway ${ }^{\mathrm{a}}$

\begin{tabular}{|c|c|c|c|c|}
\hline ID & Term & Count (\%) & P-value & Genes \\
\hline hsa04020 & $\begin{array}{l}\text { Calcium signaling } \\
\text { pathway }\end{array}$ & $30(6.03)$ & $6.86 \times 10^{-05}$ & $\begin{array}{l}\text { ADCY1, ADCY8, TACR1, DRD5, BDKRB2, } \\
\text { EDNRA, ATP2B2, EDNRB, HRH1 and } \\
\text { PDE1C }\end{array}$ \\
\hline hsa00140 & $\begin{array}{l}\text { Steroid hormone } \\
\text { biosynthesis }\end{array}$ & $15(7.03)$ & $7.84 \times 10^{-05}$ & $\begin{array}{l}\text { CYP3A4, STS, CYP1B1, CYP11A1, CYP2E1, } \\
\text { UGT1A1, UGT1A9, UGT2B17, UGT1A8 and } \\
\text { AKR1C4 }\end{array}$ \\
\hline hsa00980 & $\begin{array}{l}\text { Metabolism of xenobi } \\
\text { otics by cytochrome } \\
\text { P450 }\end{array}$ & 17 (8.03) & $1.03 \times 10^{-04}$ & $\begin{array}{l}\text { CYP3A4, CYP1B1, CYP2F1, SULT2A1, } \\
\text { CYP2B6, ADH1C, CYP2E1, UGT1A1, } \\
\text { ALDH3A1 and UGT1A9 }\end{array}$ \\
\hline hsa04060 & $\begin{array}{l}\text { Cytokine-cytokine } \\
\text { receptor interaction }\end{array}$ & $35(9.03)$ & $1.12 \times 10^{-04}$ & $\begin{array}{l}\text { IFNA21, CSF2, KITLG, TNFSF14, CXCR2, } \\
\text { IL15, CCL5, CXCL11, CCL4 and TGFB2 }\end{array}$ \\
\hline
\end{tabular}

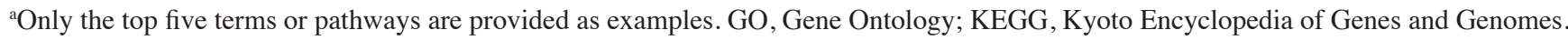

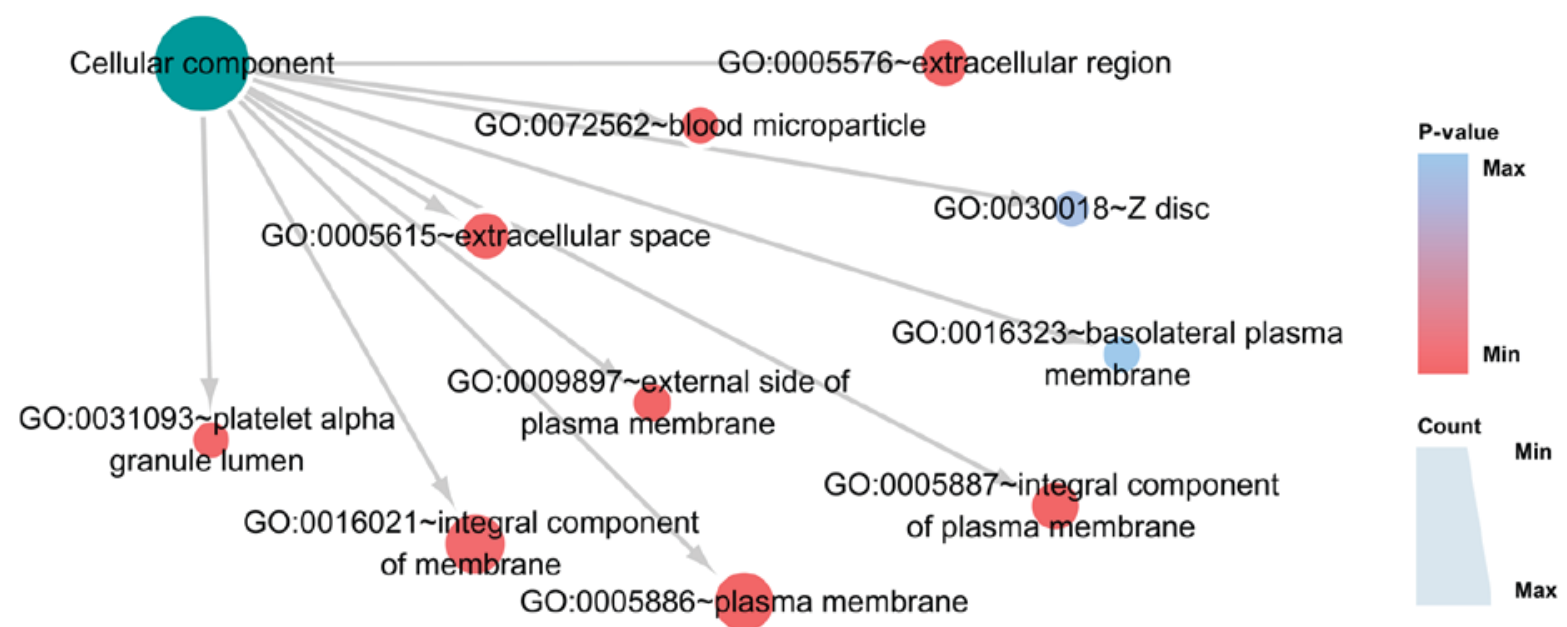

Figure 9. Cellular component of differentially expressed genes in MCF-7 cells treated with cinobufotalin. The circles represent different GO terms. The colors of circles indicate the significance of the corresponding terms, and the sizes of circles display the numbers of genes enriched in this term. GO, Gene Ontology.

trichostatin A (appearing twice), esculetin, metixene, niclosamide, 15-delta prostaglandin $\mathrm{J} 2$, pimethixene, acetohexamide, allantoin and pregnenolone. The 1,237 DEGs generated after cinobufotalin treatment in $\mathrm{MCF}-7$ cells were also compared with the genes following treatment with other drugs from the CMAP project in MCF-7 and PC3 cell lines. A total of 11 compounds were chosen with $\mathrm{P}<0.05$. The results revealed that the compounds exhibiting similar roles to cinobufotalin included miconazole, salbutamol, dexibuprofen, ciprofloxacin, nialamide, scopolamine $\mathrm{N}$-oxide and cinnarizine, whereas those exhibiting an opposite effect included triamterene, iloprost, BCB000040 and BCB000038 (Table III; Fig. 12). Among these drugs, most of them were generated from the same cell line, MCF-7, which enhanced the power of drug prediction, e.g. miconazole, salbutamol, iloprost, dexibuprofen, ciprofloxacin, nialamide, scopolamine N-oxide and cinnarizine may have comparable target genes with cinobufotalin in breast cancer cells.

\section{Discussion}

Currently, to the best of the authors' knowledge, there have been no reports on the antitumor mechanisms of cinobufotalin in breast cancer cells through large data mining analyses. Microarrays and RNA-sequencing have facilitated research on functions and mechanisms of TCM (49-52). The present study was conducted by combining microarray analysis and RNA-sequencing data in breast cancer tissues. For the potential target gene of cinobufotalin, several genes were selected for confirmation and demonstrated that ALB, FYN, TF, SRC and CDKN2A may serve pivotal roles in the onset and development of breast cancer. These genes also were affected by cinobufotalin in treated MCF-7 cells, which may shed light on the potential mechanism of cinobufotalin on breast cancer cells. In the present study, the pathways of 'neuroactive ligand-receptor interaction' and 'calcium signaling' appeared to be significant pathways for cinobufotalin in MCF-7 breast 


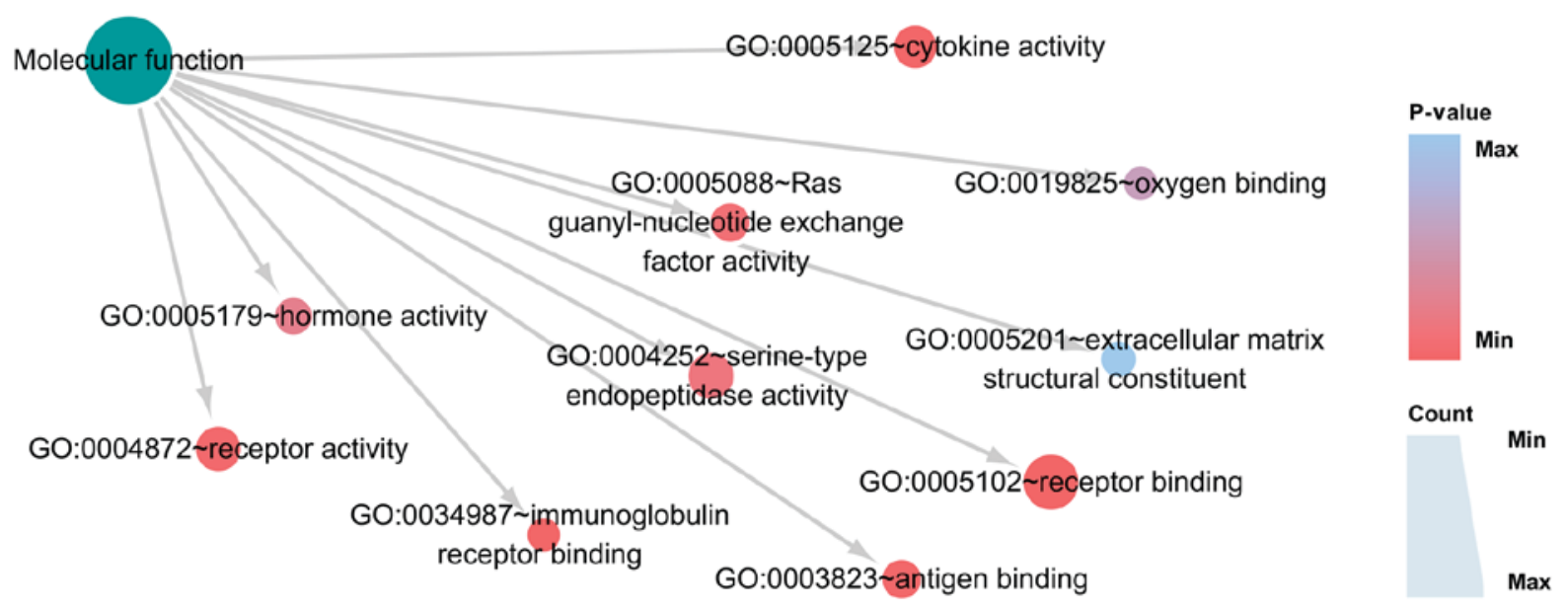

Figure 10. Molecular function of differentially expressed genes in MCF-7 cells treated with cinobufotalin. The circles represent different GO terms. The colors of circles indicate the significance of the corresponding terms, and the sizes of circles display the numbers of genes enriched in this term. GO, Gene Ontology.

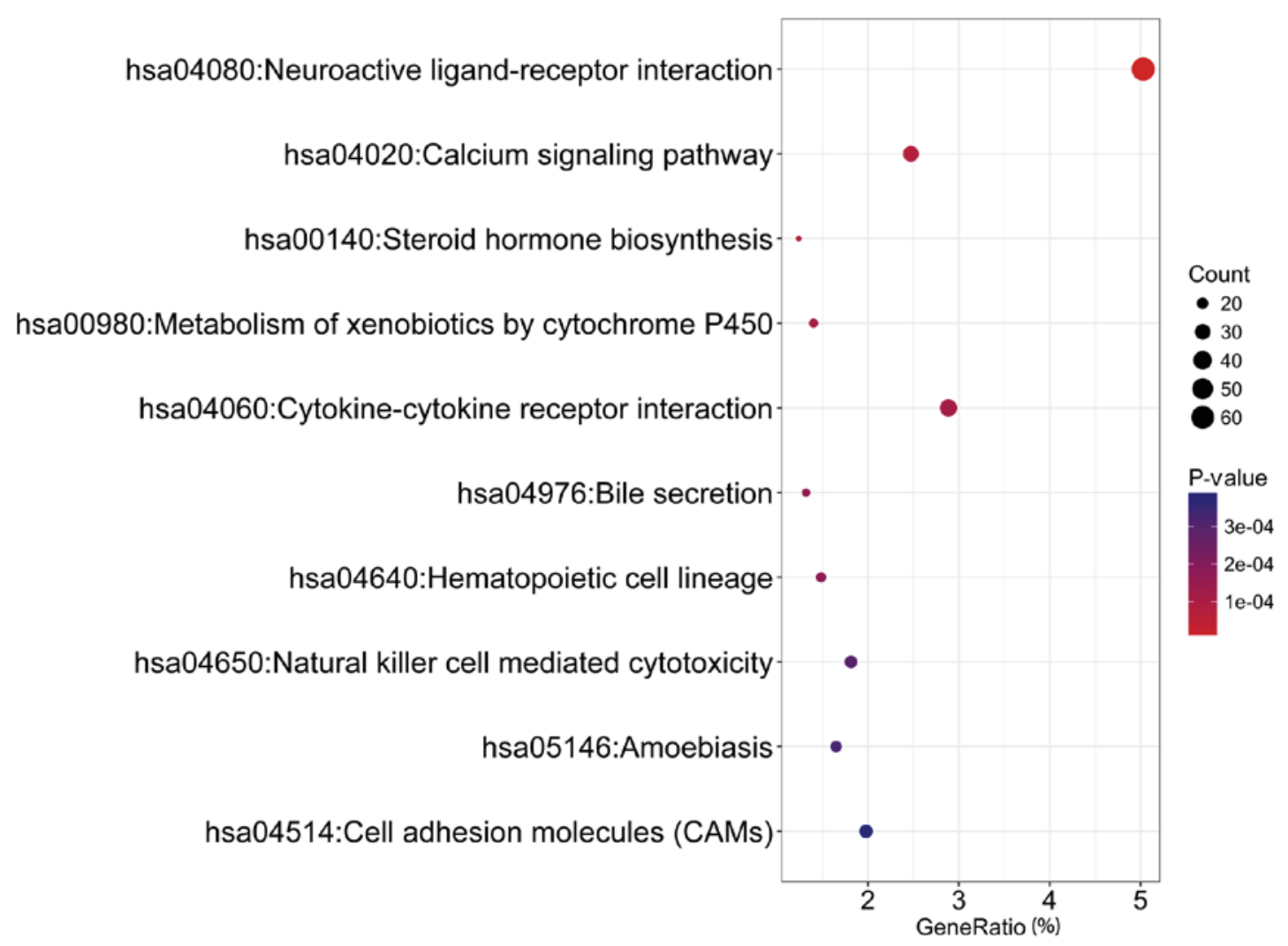

Figure 11. Kyoto Encyclopedia of Genes and Genomes pathways of differentially expressed genes in MCF-7 cells treated with cinobufotalin. Circle color indicates the significance of the corresponding terms, and circle size represents the number of genes enriched in this term.

cancer cells. In addition, connectivity mapping demonstrated that cinobufotalin had similar molecular mechanisms to drugs such as miconazole as they target consistent genes in breast cancer cells, which may provide a theoretical foundation for research on the anticancer mechanism of cinobufotalin in breast cancer cells.

The anticancer ability of cinobufotalin has been previously documented in hepatoblastoma (24) and lung cancer cells (25), based on in vitro models. In HepG2 hepatoblastoma cells, cinobufotalin was reported to inactivate Akt-S6K1 signaling, and in A549, H460 and HTB-58 lung cancer cells, cinobufotalin mainly induced Cyclophilin D-dependent non-apoptotic death. Data from PubChem also revealed that cinobufotalin exhibited effects on other cancer cells. For instance, cinobufotalin cytotoxicity against human Bel7402 cells, which have been identified as being derived from Hela cells, was detected by MTT assay (BioAssay AID: 343717) and the activity value $\left(\mathrm{IC}_{50}\right)$ was $1.21 \mathrm{mM}$. Another BioAssay (AID: 1221865) indicated an activity value (IC 50) of $8.62 \mathrm{mM}$ when cytotoxicity against human Bel7402 cells was assessed after treatment of $72 \mathrm{~h}$. Interestingly, a phase I clinical trial sponsored by Shanghai University of TCM is now at 
Table II. Total rank of the compounds from CMAP analysis compared with cinobufotalin in MCF-7 breast cancer cells.

\begin{tabular}{|c|c|c|c|c|}
\hline Rank & Batch from CMAP & Compound name & Dose & Connectivity score \\
\hline 1 & 627 & Trichostatin A & $100 \mathrm{nM}$ & 1.00 \\
\hline 2 & 772 & Esculetin & $22 \mu \mathrm{M}$ & 1.00 \\
\hline 3 & 655 & Metixene & $12 \mu \mathrm{M}$ & 0.99 \\
\hline 4 & 632 & Niclosamide & $12 \mu \mathrm{M}$ & 0.99 \\
\hline 5 & 655 & Trichostatin A & $100 \mathrm{nM}$ & 0.99 \\
\hline 6 & 765 & 15-delta prostaglandin $\mathrm{J} 2$ & $10 \mu \mathrm{M}$ & 0.98 \\
\hline 7 & 772 & Pimethixene & $10 \mu \mathrm{M}$ & 0.97 \\
\hline 8 & 772 & Acetohexamide & $12 \mu \mathrm{M}$ & 0.97 \\
\hline 9 & 627 & Allantoin & $25 \mu \mathrm{M}$ & 0.97 \\
\hline 10 & 656 & Pregnenolone & $13 \mu \mathrm{M}$ & 0.96 \\
\hline
\end{tabular}

CMAP, Connectivity Map database. Trichostatin A appeared twice.

Table III. Rank of the compounds from CMAP analysis compared with cinobufotalin in a single cell line.

\begin{tabular}{rllcr}
\hline Rank & Compound name & Cell line & Enrichment & P-value \\
\hline 1 & Miconazole & MCF-7 & 0.966 & 0.002 \\
2 & Triamterene & PC3 & -0.964 & 0.003 \\
3 & Salbutamol & MCF-7 & 0.953 & 0.004 \\
4 & Iloprost & MCF-7 & -0.937 & 0.008 \\
5 & BCB000040 & PC3 & -0.929 & 0.010 \\
6 & Dexibuprofen & MCF-7 & 0.920 & 0.013 \\
7 & BCB000038 & PC3 & -0.908 & 0.017 \\
8 & Ciprofloxacin & MCF-7 & 0.906 & 0.018 \\
9 & Nialamide & MCF-7 & 0.904 & 0.019 \\
10 & Scopolamine N-oxide & MCF-7 & 0.888 & 0.026 \\
11 & Cinnarizine & MCF-7 & 0.872 & 0.033 \\
\hline
\end{tabular}

CMAP, Connectivity Map database.

recruitment stage and will use cinobufotalin injection as intervention to treat malignant tumor of small intestine metastatic to liver (https://clinicaltrials.gov; ClinicalTrials.gov Identifier: NCT03189992). However, no previous study has examined the effects and mechanism of cinobufotalin on breast cancer cells. From the expression data provided by the GSE85871 data set in MCF-7 cells following cinobufotalin treatment (26), the present study identified 1,237 DEGs, and subsequently conducted further analysis of the core genes disclosed by PPI. Additional analysis demonstrated that some of these core genes, to some extent, may influence the onset and development of breast cancer through their abnormal expression and genetic alteration. According to the data in TCGA and The Human Protein Atlas, the mRNA and protein expression levels of SRC and CDKN2A were increased in breast cancer tissues compared with non-cancerous tissues. Previous studies have also reported that increased SRC and CDKN2 expression levels correlated with the onset, metastasis and prognosis of breast cancer (53-59). Therefore, SRC and CDKN2 may be the most important hub genes in the biological function of cinobufotalin on breast cancer MCF-7 cells, as in this study, cinobufotalin was observed to inhibit the overexpression of SRC and CDKN2A (data not shown). The potential targeting of these genes suggested that cinobufotalin may have anticancer potential.

In addition, potential mechanisms of cinobufotalin in MCF-7 breast cancer cells were elucidated from the prospective signaling pathways. The 1,237 DEGs were annotated to examine how cinobufotalin functioned on breast cancer cells. Notably, the GO term results of the 1,237 DEGs were mainly linked to immunity, including 'immune response', 'regulation of immune response', 'innate immune response' and 'adaptive immune response'. It was reported previously that cinobufotalin activated the nuclear factor- $\kappa \mathrm{B}$ pathway and decreased expression levels of brain-derived neurotrophic factor to induce neuroinflammation in rats (60). Nonetheless, no direct evidence has revealed that cinobufotalin was associated with number of immune cells, organism immunity or tumor 
A

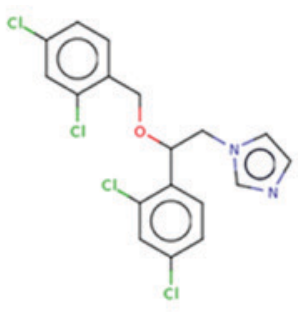

C<smiles>CC(C)Cc1ccc(N(C)C(=O)O)cc1</smiles>

E<smiles>O=C(CCNNC(=O)c1ccncc1)NCc1ccccc1</smiles>

G<smiles>C(=C/c1ccccc1)\CN1CCCN(C(c2ccccc2)c2ccccc2)CC1</smiles>

I<smiles>CC#CCC(C)N(C)C=CN1CN2CC(=CCCCC(=O)O)CN2C1</smiles>

\section{K}<smiles>O=C(O)CCCCCC(=O)N/N=C/c1cccs1</smiles>

B<smiles>CC(C)(C)NCC(O)c1ccc(O)c(CO)c1</smiles><smiles>O=C(O)c1cn(C2CC2)c2cc(N3CCNCC3)c(F)cc2c1=O</smiles>

$\mathbf{F}$<smiles>C[N+]([O-])(O)CN1CNN(OC(=O)N(CO)c2ccccc2)C1</smiles>

H<smiles>Nc1nc(N)c2nc(-c3ccccc3)c(N)nc2n1</smiles>

J

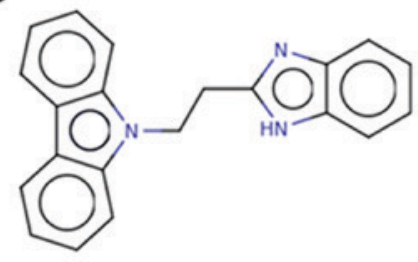

Figure 12. Chemical structures of 11 compounds acquired from the CMAP database. (A) Miconazole. (B) Salbutamol. (C) Dexibuprofen. (D) Ciprofloxacin. (E) Nialamide. (F) Scopolamine N-oxide. (G) Cinnarizine. (H) Triamterene. (I) Iloprost. (J) BCB000040. (K) BCB000038. CMAP, Connectivity Map database.

immunity. On a molecular level, the present study hypothesized that in treating breast cancer, cinobufotalin may exert its antitumor influences by activating the immune response; however, additional studies are required for verification.

To further interpret the potential mechanism of cinobufotalin in MCF-7 breast cancer cells, KEGG pathway enrichment was performed on the identified DEGs, which revealed that several pathways were connected, not only to immunity after cinobufotalin was applied to treat breast cancer samples but also to other pathways. 'Neuroactive ligand-receptor interaction', which contains numerous $\mathrm{G}$ protein-coupled receptors, continued to be the most significantly enriched pathway. As one of the most common pathways of malignancies, the pathway of 'Neuroactive ligand-receptor interaction' ranks fifth in genes with mutation in the central nervous system (61). A previous study reported that low expression of cannabinoid receptor-1 (CNR1), part of the neuroactive ligand-receptor interaction pathway, indicated that breast cancer patients may benefit from chemotherapy (62). Notably, this CNR1 was among the 1,237 DEGs following cinobufotalin treatment in breast cancer cells. In addition, this pathway was reported to serve a vital role in tumorigenesis and chemotherapy for breast cancer $(62,63)$, 
which suggested that cinobufotalin may exhibit its curative potential for breast cancer by influencing the neuroactive ligand-receptor interaction pathway. The co-treatment of cinobufotalin to chemotherapeutics may induce a synergistic effect by suppressing the neuroactive ligand-receptor interaction pathway.

The second most enriched KEGG pathway was the 'calcium signaling pathway', the mechanism of which is rather complicated in breast cancer. Previous studies have reported that the calcium signaling pathway interacts with other pathways to contribute to the onset and progression of breast cancer (64-68). In invasive ductal carcinoma the calcium signaling pathway was reported to interact with pathways in cancer, including the pathways of glyoxylate and dicarboxylate metabolism, basal transcription factors, tyrosine metabolism, Fc $\gamma \mathrm{R}$-mediated phagocytosis, metabolism of xenobiotics by cytochrome P450 and phagosome (69). Wnt5a in the noncanonical Wnt pathway was considered a possible anti-oncogene in breast cancer, as it was demonstrated to serve an essential role via the calcium signaling pathway (70). In addition, the calcium signaling pathway was strongly linked with the proliferation, migration and invasion of breast cancer cells (71). Based on these results, cinobufotalin may affect breast cancer cells by inhibiting the calcium signaling pathway.

TCMs differ from other chemical compounds owing to their natural ingredients and low toxicity (72-75). CMAP analysis revealed that cinobufotalin and miconazole shared similar mechanisms, as the varied genes post-miconazole treatment were comparable to those following cinobufotalin treatment in the same breast cancer cell line MCF-7. It was previously demonstrated that miconazole was able to induce apoptosis in bladder cancer cells through the death receptor 5-dependent and mitochondrial-mediated pathways (76); thus, cinobufotalin may also cause the death of cancer cells $(24,25)$. Notably, miconazole activates the release of phospholipase $\mathrm{C}$-dependent $\mathrm{Ca}^{2+}$ from the endoplasmic reticulum by influencing the elevation of calcium ions, thus inducing ZR-75-1 breast cancer cell apoptosis (77). Based on these previous studies and the present CMAP analysis, it was hypothesized that cinobufotalin may induce the apoptosis of cancer cells in breast cancer MCF-7 cells via the calcium signaling pathway, thus resembling the curative properties of miconazole. However, this hypothesis requires additional experiments for confirmation.

Several limitations exist in the present study. First, the cellular model MCF-7 only represents a specific subtype of breast cancer. It is probable that cinobufotalin may serve different functions through various genes and pathways in distinct subtypes of breast cancer. Therefore, additional cell lines should be examined in future studies. Second, the current findings are based on in silico analyses and verification with in vitro and in vivo experiments are needed, including the biological effect and potential molecular mechanism.

In conclusion, cinobufotalin is likely to act as an effective compound to treat this subtype of breast cancer, triple positive for estrogen, progesterone and HER-2 receptors, and its mechanism may correlate to various pathways. In addition, cinobufotalin may have anticancer functions in MCF-7 cells similar to those of miconazole.

\section{Acknowledgements}

The authors are grateful for the public microarray and RNA-sequencing data.

\section{Funding}

The present study was supported by The Guangxi Provincial Health Bureau Scientific Research Project (grant no. Z2013424).

\section{Availability of data and materials}

All data generated or analyzed during this study are included in this published article.

\section{Authors' contributions}

JL, MHR, YWD, RQH, JCZ, JM and GC designed the study. JL, MHR, XJL, DDX, LJZ, HQ, CXF and GC conceived and performed the experiments. JL, MHR, XJL, DDX, LJZ, HQ, CXF, QL and HY performed the statistical analysis and data interpretation. JL, MHR and RQH wrote the manuscript, HY, JCZ and GC corrected the paper. All authors approved the final version of the manuscript for publication.

\section{Ethics approval and consent to participate}

Not applicable.

\section{Patient consent for publication}

Not applicable.

\section{Competing interests}

The authors declare that they have no competing interests.

\section{References}

1. Kolak A, Kamińska M, Sygit K, Budny A, Surdyka D, Kukielka-Budny B and Burdan F: Primary and secondary prevention of breast cancer. Ann Agric Environ Med 24: 549-553, 2017.

2. Yedjou CG, Tchounwou PB, Payton M, Miele L, Fonseca DD, Lowe L and Alo RA: Assessing the racial and ethnic disparities in breast cancer mortality in the United States. Int J Environ Res Public Health 14: E486, 2017.

3. Wang W, Song XW and Zhao CH: Roles of programmed cell death protein 5 in inflammation and cancer (Review). Int J Oncol 49: 1801-1806, 2016.

4. Pan Z, Jing W, He K, Zhang L and Long X: SATB1 is correlated with progression and metastasis of breast cancers: A meta-analysis. Cell Physiol Biochem 38: 1975-1983, 2016.

5. Wang M, Zhang C, Song Y, Wang Z, Wang Y, Luo F, Xu Y, Zhao Y, Wu Z and Xu Y: Mechanism of immune evasion in breast cancer. Onco Targets Ther 10: 1561-1573, 2017.

6. Liu H, Chen Y, Wu S, Song F, Zhang H and Tian M: Molecular imaging using PET and SPECT for identification of breast cancer subtypes. Nucl Med Commun 37: 1116-1124, 2016.

7. Xing L, He Q, Wang YY, Li HY and Ren GS: Advances in the surgical treatment of breast cancer. Chin Clin Oncol 5: 34, 2016.

8. De A, Kuppusamy G and Karri VVSR: Affibody molecules for molecular imaging and targeted drug delivery in the management of breast cancer. Int J Biol Macromol 107: 906-919, 2018 
9. Matos Do Canto L, Marian C, Varghese RS, Ahn J, Da Cunha PA, Willey S, Sidawy M, Rone JD, Cheema AK, Luta G, et al: Metabolomic profiling of breast tumors using ductal fluid. Int J Oncol 49: 2245-2254, 2016

10. Quispe-Soto ET and Calaf GM: Effect of curcumin and paclitaxel on breast carcinogenesis. Int J Oncol 49: 2569-2577, 2016.

11. Yang N, Zhou TC, Lei XX, Wang C, Yan M, Wang ZF, Liu W, Wang J, Ming KH, Wang BC, et al: Inhibition of Sonic Hedgehog signaling pathway by thiazole antibiotic thiostrepton attenuates the CD44+/CD24-stem-like population and sphere-forming capacity in triple-negative breast cancer. Cell Physiol Biochem 38: 1157-1170, 2016.

12. Canfeld K, Wells W, Geradts J, Kinlaw WB, Cheng C and Kurokawa M: Inverse association between MDM2 and HUWE1 protein expression levels in human breast cancer and liposarcoma. Int J Clin Exp Pathol 9: 6342-6349, 2016.

13. Santa Mina D, Brahmbhatt P, Lopez C, Baima J, Gillis C, Trachtenberg L and Silver JK: The case for prehabilitation prior to breast cancer treatment. PM R 9 (Suppl): S305-S316, 2017.

14. Mo CH, Gao L, Zhu XF, Wei KL, Zeng JJ, Chen G and Feng ZB The clinicopathological significance of UBE2C in breast cancer: A study based on immunohistochemistry, microarray and RNA-sequencing data. Cancer Cell Int 17: 83, 2017.

15. Gomulkiewicz A, Jablonska K, Pula B, Grzegrzolka J, Borska S, Podhorska-Okolow M, Wojnar A, Rys J, Ambicka A, Ugorski M, et al: Expression of metallothionein 3 in ductal breast cancer. Int J Oncol 49: 2487-2497, 2016.

16. Xiong DD, Lv J, Wei KL, Feng ZB, Chen JT, Liu KC, Chen G and Luo DZ: A nine-miRNA signature as a potential diagnostic marker for breast carcinoma: An integrated study of 1,110 cases. Oncol Rep 37: 3297-3304, 2017.

17. Shen SQ, Huang LS, Xiao XL, Zhu XF, Xiong DD, Cao XM, Wei KL, Chen G and Feng ZB: miR-204 regulates the biological behavior of breast cancer MCF-7 cells by directly targeting FOXA1. Oncol Rep 38: 368-376, 2017.

18. Li CY, Xiong DD, Huang CQ, He RQ, Liang HW, Pan DH, Wang HL, Wang YW, Zhu HW and Chen G: Clinical value of miR-101-3p and biological analysis of its prospective targets in breast cancer: A study based on The Cancer Genome Atlas (TCGA) and bioinformatics. Med Sci Monit 23: 1857-1871, 2017.

19. Efferth T, Li PC, Konkimalla VS and Kaina B: From traditional Chinese medicine to rational cancer therapy. Trends Mol Med 13: 353-361, 2007

20. Wang S, Penchala S, Prabhu S, Wang J and Huang Y: Molecular basis of traditional Chinese medicine in cancer chemoprevention. Curr Drug Discov Technol 7: 67-75, 2010.

21. Qi F, Li A, Zhao L, Xu H, Inagaki Y, Wang D, Cui X, Gao B, Kokudo N, Nakata M and Tang W: Cinobufacini, an aqueous extract from Bufo bufo gargarizans Cantor, induces apoptosis through a mitochondria-mediated pathway in human hepatocellular carcinoma cells. J Ethnopharmacol 128: 654-661, 2010.

22. Yin PH, Liu X, Qiu YY, Cai JF, Qin JM, Zhu HR and Li Q: Anti-tumor activity and apoptosis-regulation mechanisms of bufalin in various cancers: New hope for cancer patients. Asian Pac J Cancer Prev 13: 5339-5343, 2012.

23. Chen KK, Anderson RC and Henderson FG: Comparison of cardiac action of bufalin, cinobufotalin, and telocinobufagin with cinobufagin. Proc Soc Exp Biol Med 76: 372-374, 1951.

24. Cheng L, Chen YZ, Peng Y, Yi N, Gu XS, Jin Y and Bai XM: Ceramide production mediates cinobufotalin-induced growth inhibition and apoptosis in cultured hepatocellular carcinoma cells. Tumour Biol 36: 5763-5771, 2015.

25. Kai S, Lu JH, Hui PP and Zhao H: Pre-clinical evaluation of cinobufotalin as a potential anti-lung cancer agent. Biochem Biophys Res Commun 452: 768-774, 2014.

26. Lv C, Wu X, Wang X, Su J, Zeng H, Zhao J, Lin S, Liu R, Li H, $\mathrm{Li} \mathrm{X}$ and Zhang W: The gene expression profiles in response to 102 traditional Chinese medicine (TCM) components: A general template for research on TCMs. Sci Rep 7: 352, 2017.

27. Gao J, Aksoy BA, Dogrusoz U, Dresdner G, Gross B, Sumer SO, Sun Y, Jacobsen A, Sinha R, Larsson E, et al: Integrative analysis of complex cancer genomics and clinical profiles using the cBioPortal. Sci Signal 6: pl1, 2013.

28. Harryman WL, Pond E, Singh P, Little AS, Eschbacher JM, Nagle RB and Cress AE: Laminin-binding integrin gene copy number alterations in distinct epithelial-type cancers. Am J Transl Res 8: 940-954, 2016.

29. Tang Z, Li C, Kang B, Gao G, Li C and Zhang Z: GEPIA: A web server for cancer and normal gene expression profiling and interactive analyses. Nucleic Acids Res 45: W98-W102, 2017.
30. Thul PJ and Lindskog C: The human protein atlas: A spatial map of the human proteome. Protein Sci 27: 233-244, 2018.

31. He RQ, Li XJ, Liang L, Xie Y, Luo DZ, Ma J, Peng ZG, Hu XH and Chen G: The suppressive role of miR-542-5p in NSCLC: The evidence from clinical data and in vivo validation using a chick chorioallantoic membrane model. BMC Cancer 17: 655, 2017.

32. Uhlen M, Zhang C, Lee S, Sjöstedt E, Fagerberg L, Bidkhori G, Benfeitas R, Arif M, Liu Z, Edfors F, et al: A pathology atlas of the human cancer transcriptome. Science 357: pii: eaan2507, 2017.

33. Panosyan EH, Lin HJ, Koster J and Lasky JL III: In search of druggable targets for GBM amino acid metabolism. BMC Cancer 17: 162, 2017.

34. Petrizzo A, Caruso FP, Tagliamonte M, Tornesello ML, Ceccarelli M, Costa V, Aprile M, Esposito R, Ciliberto G, Buonaguro FM and Buonaguro L: Identification and validation of HCC-specific gene transcriptional signature for tumor antigen discovery. Sci Rep 6: 29258, 2016.

35. Xu X, Wang X, Fu B, Meng L and Lang B: Differentially expressed genes and microRNAs in bladder carcinoma cell line 5637 and T24 detected by RNA sequencing. Int J Clin Exp Pathol 8: 12678-12687, 2015.

36. Cao L, Zhang Q, Cheng S, Chen Z, Hua Z, Yang J, Liu D and Cui N: Long non-coding RNAs and genes contributing to the generation of cancer stem cells in hepatocellular carcinoma identified by RNA sequencing analysis. Oncol Rep 36: 2619-2624, 2016.

37. Wang DW, Yu SY, Cao Y, Yang L, Liu W, Er XQ, Yao GJ and Bi ZG: Identification of CD20, ECM, and ITGA as biomarkers for osteosarcoma by integrating transcriptome analysis. Med Sci Monit 22: 2075-2085, 2016

38. $\mathrm{Xu} \mathrm{X}$ and $\mathrm{Li} \mathrm{H}$ : Integrated microRNA-gene analysis of coronary artery disease based on miRNA and gene expression profiles. Mol Med Rep 13: 3063-3073, 2016.

39. Wang Y, Zhang J, Li L, Xu X, Zhang Y, Teng Z and Wu F: Identification of molecular targets for predicting colon adenocarcinoma. Med Sci Monit 22: 460-468, 2016.

40. Chen W, Liu Q, Lv Y, Xu D, Chen W and Yu J: Special role of JUN in papillary thyroid carcinoma based on bioinformatics analysis. World J Surg Oncol 15: 119, 2017.

41. Tian W, Liu J, Pei B, Wang X, Guo Y and Yuan L: Identification of miRNAs and differentially expressed genes in early phase non-small cell lung cancer. Oncol Rep 35: 2171-2176, 2016.

42. Li J, Yang X, Guan H, Mizokami A, Keller ET, Xu X, Liu X, Tan J, Hu L, Lu Y and Zhang J: Exosome-derived microRNAs contribute to prostate cancer chemoresistance. Int J Oncol 49: 838-846, 2016.

43. Song B, Du J, Feng Y, Gao YJ and Zhao JS: Co-expressed differentially expressed genes and long non-coding RNAs involved in the celecoxib treatment of gastric cancer: An RNA sequencing analysis. Exp Ther Med 12: 2455-2468, 2016.

44. Zhang H, Zhang X, Huang J and Fan X: Identification of key genes and pathways for peri-implantitis through the analysis of gene expression data. Exp Ther Med 13: 1832-1840, 2017.

45. Yu H, Pei D, Chen L, Zhou X and Zhu H: Identification of key genes and molecular mechanisms associated with dedifferentiated liposarcoma based on bioinformatic methods. Onco Targets Ther 10: 3017-3027, 2017.

46. Nicolau CA, Prorock A, Bao Y, Neves-Ferreira AGDC, Valente RH and Fox JW: Revisiting the therapeutic potential of Bothrops jararaca venom: Screening for novel activities using connectivity mapping. Toxins (Basel) 10: E69, 2018

47. Thillaiyampalam G, Liberante F, Murray L, Cardwell C, Mills K and Zhang SD: An integrated meta-analysis approach to identifying medications with potential to alter breast cancer risk through connectivity mapping. BMC Bioinformatics 18: 581 , 2017.

48. Busby J, Murray L, Mills K, Zhang SD, Liberante F and Cardwell CR: A combined connectivity mapping and pharmacoepidemiology approach to identify existing medications with breast cancer causing or preventing properties Pharmacoepidemiol Drug Saf 27: 78-86, 2018.

49. Liu Y, Zhao Y, Wei Z, Tao L, Sheng X, Wang S, Chen J, Ruan J, Liu Z, Cao Y, et al: Targeting thioredoxin system with an organosulfur compound, diallyl trisulfide (DATS), attenuates progression and metastasis of triple-negative breast cancer (TNBC). Cell Physiol Biochem 50: 1945-1963, 2018.

50. Liao KF, Chiu TL, Huang SY, Hsieh TF, Chang SF, Ruan JW, Chen SP, Pang CY and Chiu SC: Anti-cancer effects of radix angelica sinensis (Danggui) and N-butylidenephthalide on gastric cancer: Implications for REDD1 activation and mTOR inhibition. Cell Physiol Biochem 48: 2231-2246, 2018. 
51. Hao J, Jin Z, Zhu H, Liu X, Mao Y, Yang X, Gao L, Liu D, Chen D and Wu X: Antiestrogenic activity of the Xi-huang formula for breast cancer by targeting the estrogen receptor $\alpha$. Cell Physiol Biochem 47: 2199-2215, 2018.

52. Ding X, Yang Q, Kong X, Haffty BG, Gao S and Moran MS Radiosensitization effect of Huaier on breast cancer cells. Oncol Rep 35: 2843-2850, 2016.

53. Browne AL, Charmsaz S, Vareslija D, Fagan A, Cosgrove N Cocchiglia S, Purcell S, Ward E, Bane F, Hudson L, et al: Network analysis of SRC-1 reveals a novel transcription factor hub which regulates endocrine resistant breast cancer. Oncogene 37: 2008-2021, 2018.

54. Hamurcu Z, Delibasi N, Gecene S, Sener EF, Dönmez-Altuntas H, Ozkul Y, Canatan H and Ozpolat B: Targeting LC3 and Beclin-1 autophagy genes suppresses proliferation, survival, migration and invasion by inhibition of Cyclin-D1 and uPAR/Integrin $\beta 1 / \mathrm{Src}$ signaling in triple negative breast cancer cells. J Cancer Res Clin Oncol 144: 415-430, 2018.

55. Abdullah C, Korkaya H, Iizuka S and Courtneidge SA: SRC increases MYC mRNA expression in $\mathrm{ER}^{+}$breast cancer via mRNA stabilization and inhibition of p53 function. Mol Cell Biol: pii: MCB.00463-17, 2017 (Epub ahead of print).

56. Ocana A, Gil-Martin M, Martin M, Rojo F, Antolin S, Guerrero A, Trigo JM, Munoz M, Pandiella A, et al: A phase I study of the SRC kinase inhibitor dasatinib with trastuzumab and paclitaxel as first line therapy for patients with HER2-overexpressing advanced breast cancer. GEICAM/2010-04 study. Oncotarget 8: 73144-73153, 2017.

57. Li Y, Zhou W, Tang K, Chen X, Feng Z and Chen J: Silencing Aurora A leads to re-sensitization of breast cancer cells to Taxol through downregulation of SRC-mediated ERK and mTOR pathways. Oncol Rep 38: 2011-2022, 2017.

58. Subash-Babu P, Li DK and Alshatwi AA: In vitro cytotoxic potential of friedelin in human MCF-7 breast cancer cell: Regulate early expression of Cdkn2a and pRb1, neutralize mdm2-p53 amalgamation and functional stabilization of p53. Exp Toxicol Pathol 69: 630-636, 2017.

59. Spitzwieser M, Entfellner E, Werner B, Pulverer W, Pfeiler G, Hacker S and Cichna-Mark1 M: Hypermethylation of CDKN2A exon 2 in tumor, tumor-adjacent and tumor-distant tissues from breast cancer patients. BMC Cancer 17: 260, 2017.

60. Bi QR, Hou JJ, Qi P, Ma CH, Shen Y, Feng RH, Yan BP, Wang JW, Shi XJ, Zheng YY, et al: Venenum Bufonis induces rat neuroinflammation by activiating NF- $\mathrm{B}$ pathway and attenuation of BDNF. J Ethnopharmacol 186: 103-110, 2016.

61. Engel SR and Grant KA: Neurosteroids and behavior. Int Rev Neurobiol 46: 321-348, 2001

62. Li Y, Liu X, Tang H, Yang H and Meng X: RNA sequencing uncovers molecular mechanisms underlying pathological complete response to chemotherapy in patients with operable breast cancer. Med Sci Monit 23: 4321-4327, 2017.

63. Zhou L, Gao HF, Liu DS, Feng JY, Gao DD and Xia W: Gene expression profiling of brain metastatic cell from triple negative breast cancer: Understanding the molecular events. Gene 640 21-27, 2018

64. Choi HY, Park N, Na JB, Ko ES, Park JY and Yoo JC: Direct binding of Copine3 with Jab1 activates downstream ErbB2 signaling and motility in SKBr3 breast cancer cells. Oncol Rep 35: 1147-1152, 2016.
65. Li Y, Liu X, Tang H, Yang $\mathrm{H}$ and Meng X: RNA sequencing uncovers molecular mechanisms underlying pathological complete response to chemotherapy in patients with operable breast cancer. Med Sci Monit 23: 4321-4327, 2017.

66. Yang M, Li H, Li Y, Ruan Y and Quan C: Identification of genes and pathways associated with MDR in MCF-7/MDR breast cancer cells by RNA-seq analysis. Mol Med Rep 17: 6211-6226, 2018.

67. Dang YW, Lin P, Liu LM, He RQ, Zhang LJ, Peng ZG, Li XJ and Chen G: In silico analysis of the potential mechanism of telocinobufagin on breast cancer MCF-7 cells. Pathol Res Pract 214: 631-643, 2018

68. Varga K, Hollósi A, Pászty K, Hegedûs L, Szakács G, Tímár J, Papp B, Enyedi Á and Padányi R: Expression of calcium pumps is differentially regulated by histone deacetylase inhibitors and estrogen receptor alpha in breast cancer cells. BMC Cancer 18: 1029,2018

69. Chen WY, Wu F, You ZY, Zhang ZM, Guo YL and Zhong LX Analyzing the differentially expressed genes and pathway cross-talk in aggressive breast cancer. J Obstet Gynaecol Res 41 : 132-140, 2015

70. Trifa F, Karray-Chouayekh S, Jmal E, Jmaa ZB, Khabir A, Sellami-Boudawara T, Frikha M, Daoud J and Mokdad-Gargouri R: Loss of WIF-1 and Wnt5a expression is related to aggressiveness of sporadic breast cancer in Tunisian patients. Tumour Biol 34: 1625-1633, 2013.

71. Di J, Huang H, Qu D, Tang J, Cao W, Lu Z, Cheng Q, Yang J, Bai J, Zhang Y and Zheng J: Rap2B promotes proliferation, migration, and invasion of human breast cancer through calcium-related ERK1/2 signaling pathway. Sci Rep 5: 12363, 2015.

72. Lou JS, Yao P and Tsim KWK: Cancer treatment by using traditional Chinese medicine: Probing active compounds in anti-multidrug resistance during drug therapy. Curr Med Chem 25: 5128-5141, 2018.

73. Jiang Y and Gao H: Pharmacophore-based drug design for potential AChE inhibitors from traditional chinese medicine database. Bioorg Chem 76: 400-414, 2018.

74. Zheng X, Wu F, Lin X, Shen L and Feng Y: Developments in drug delivery of bioactive alkaloids derived from traditional Chinese medicine. Drug Deliv 25: 398-416, 2018.

75. Xia X, Cole SPC, Cai T and Cai Y: Effect of traditional Chinese medicine components on multidrug resistance in tumors mediated by P-glycoprotein. Oncol Lett 13: 3989-3996, 2017.

76. Yuan SY, Shiau MY, Ou YC, Huang YC, Chen CC, Cheng CL, Chiu KY, Wang SS and Tsai KJ: Miconazole induces apoptosis via the death receptor 5-dependent and mitochondrial-mediated pathways in human bladder cancer cells. Oncol Rep 37: 3606-3616, 2017.

77. Roan CJ, Chou CT, Liang WZ, Chang HT, Kuo DH, Kuo CC, Chen FA, Shieh P and Jan CR: Effect of miconazole on $\left[\mathrm{Ca}^{2+}\right]_{\text {, }}$ and cytotoxicity in ZR-75-1 human breast cancer cells. Chin J Physiol 58: 377-384, 2015.

(7) $\Theta$ This work is licensed under a Creative Commons Attribution-NonCommercial-NoDerivatives 4.0 International (CC BY-NC-ND 4.0) License. 